\title{
On the motion of a triple pendulum system under the influence of excitation force and torque
}

\author{
T. S. Amer ${ }^{1, *}$, A. A. Galal ${ }^{2}$, A. F. Abolila ${ }^{3}$ \\ ${ }^{1}$ Mathematics Dept., Faculty of Science, Tanta University, Tanta 31527, Egypt. \\ ${ }^{2,3}$ Physics and Engineering Mathematics Dept., Faculty of Engineering, Tanta University, Tanta \\ 31734, Egypt. \\ Corresponding author: tarek.saleh@science.tanta.edu.eg
}

\begin{abstract}
In this article, a nonlinear dynamical system with three degrees of freedom (DOF) consisting of multiple pendulums (MP) is investigated. The motion of this system is restricted to be in a vertical plane, in which its pivot point moves in a circular path with constant angular velocity, under the action of an external harmonic force and a moment acting perpendicular to the direction of the last arm of MP and at the suspension point respectively. Multiple scales technique (MST) among other perturbation methods is used to obtain the approximate solutions of the equations of motion up to the third approximation because it is authorizing to execute a specific analysis of the system behaviour and to realize the solvability conditions given the resonance cases. The stability of the considered dynamical model utilizing the nonlinear stability analysis approach is examined. The solutions diagrams and resonance curves are drawn to illustrate the extent of the effect of various parameters on the solutions. The importance of this work is due to its uses in human or robotic walking analysis.
\end{abstract}

Keywords: Multiple scales technique; nonlinear dynamics; resonance; stability; triple pendulum.

\section{Introduction}

It is known that the multiple pendulums (MP) consist of several blocks connected by ropes, in which its fixed point is the other end of the first rope. These blocks and ropes may be equal or different. One of MP is the triple pendulum (TP), which is widely used in practical applications like devices that constructed to observe and detect gravity waves (Plissi et al., 2000), the crankshaft of the piston (Awrejcewicz \& Kudra, 2005a), in the devices that analysis the movement of robots (Raymond \& Virgin, 2013) and others. In (Awrejcewicz et al., 2007), the authors studied both experimentally and numerically analysis of the TP, in which it is subjected to the action of physical constraints and vibrational force. The comparison between the laboratory results and the numerical ones showed a high accuracy between them. The impact of natural frequency on the plane motion of MP is investigated in (Gupta et al., 2016). The attained results were verified with the obtained ones in (Braun, 2003). The motion of a nonlinear dynamical model represented by TP is investigated in (Gupta et al., 2017). It is observed that the motion of the third pendulum has a most chaotic behaviour than the other two pendulums. The stability of 3DOF of TP as a mechanical model with some restrictions on its position is examined in (Awrejcewicz \& Kudra., 2005b) and is considered as a special case of the work in (Awrejcewicz et al., 2004). 
On the other side, dynamical pendulum models with 2 or 3 DOF have been studied in many research works such as (Awrejcewicz et al., 2016; Starosta et al., 2011; Starosta et al., 2012; Amer et al., 2016; Nayfeh, 2004; Awrejcewicz et al., 2013; Amer et al., 2018; Amer et al., 2019; El-Sabaa et al., 2020). The plane motion of nonlinear spring pendulum with 2DOF is investigated in (Awrejcewicz et $a l ., 2016$ ) for a fixed supported point under the influence of two external forces in the direction of a spring arm and its perpendicular direction. The generalization of this model is presented in (Starosta et al., 2011) when the supported point moves in a circular path, in (Starosta et al., 2012) when this point moves along a Lissajous curve and in (Amer et al., 2016) for the motion of the same point in an elliptic path. The analytic approximate solutions of these works are obtained using the multiple scales technique (MST) (Nayfeh \& Mook, 1979; Nayfeh, 2004; Vakakis et al., 2009) and the emerged resonance cases are studied.

The vibrational motion of a damped spring rigid body pendulum is investigated in (Awrejcewicz et al., 2013) for the case of a fixed pivot point. The generalization of this problem is presented in (Amer et al., 2018), (Amer et al., 2019) when the supported point moves in an elliptic path for the cases of linear and nonlinear spring and in (El-Sabaa et al., 2020) when the supported point moves in a Lissajous curve. The conditions of solvability for the steady-state solutions are obtained and the time histories of the approximate and numerical solutions are plotted in some diagrams. A different dynamical model represented by a crane consisting of a doublependulum with constant cable length was discussed (Jaafar et al., 2019).

The dynamics of the 2DOF mathematical pendulum with variable masses are studied in (Kwiatkowski et al., 2017) while the total mass of the pendulum system remains constant. It is observed that, when the mass of the second arm increases, the amplitude of oscillations of the pendulum decreases. The numerical solutions are obtained using the Mathematica package (Wolfram, 2017). A nonlinear damped spring model is investigated in (Kamińska et al., 2018) under the action of a resistance force and in the presence of a variable harmonic moment at the point of suspension. The authors studied two approaches of MST according to two-time scales and obtained the numerical results of the original system of motion.

In the present work, we are going to study the motion of a dynamical model with $3 \mathrm{DOF}$ consisting of a triple pendulum (TP) with three different lengths in which the one tip of its first arm moves in a circular path with constant angular velocity. This motion is considered under the influence of an external harmonic force in the perpendicular direction of the third arm. Moreover, a harmonic torque acts in an anticlockwise direction at the suspension point. The governing equations of motion (GEOM) are derived using Lagrange's equations of the second type and are solved analytically using MST up to the third approximation. The emerged resonances are classified and the solvability conditions are obtained. Therefore, the steady-state oscillations are investigated. The variation of the attained solutions via time and the resonance curves are represented in different plots to show the significance of the distinct parameters on the behaviour of the dynamical model. The nonlinear stability analysis approach is used to discuss the stability of the considered model. The significance of this work is due to its interesting applications in life such as improving the walking movements of humans and robots, to predict and observe the gravity waves prophesied by relativity theory.

\section{Description of the problem}

Let us consider the nonlinear dynamical motion of a TP of lengths $\ell_{1}, \ell_{2}$ and $\ell_{3}$ with centers $z_{1}, z_{2}$ and $z_{3}$ respectively, in which their masses $m_{1}, m_{2}$ and $m_{3}$ are distributed. 
The suspension point $N$ of TP is a constraint to move in a circular path, of radius $a$, in an anticlockwise direction with constant angular velocity $\Omega$. The angles $\varphi_{1}, \varphi_{2}$ and $\varphi_{3}$ are defined as the inclination of the TP rods on the vertical axis that parallel to $O Y$ the axis in which $O X$ axis has a horizontal direction, see Figure.1.

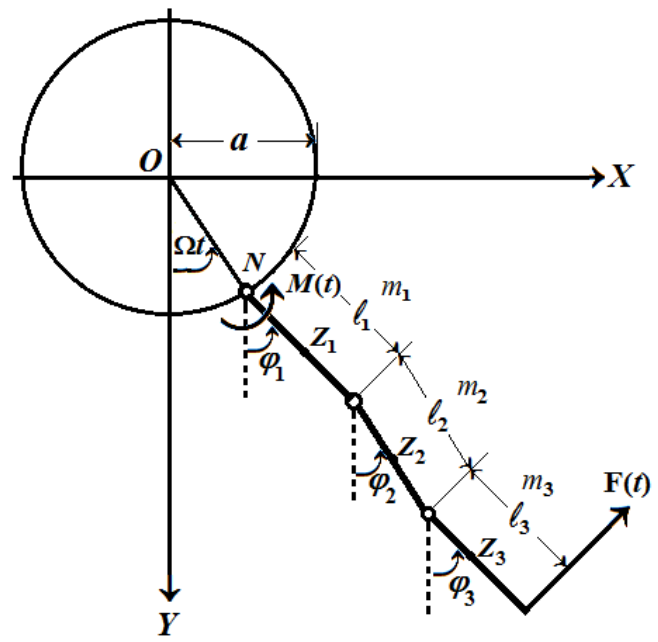

Fig.1. Triple pendulum model

The motion of the considered model under the influence of an external harmonic force acts $F(t)$ in the perpendicular direction of the bottom arm and a harmonic torque $M(t)$ at the end $N$ of the upper arm. Accordingly, we consider a planar motion of the TP and therefore, we can write the coordinates $x_{N}$ and $y_{N}$ of the point $N$ at any instance $t$ in the form:

$x_{N}=a \sin (\Omega t), \quad y_{N}=a \cos (\Omega t)$.

Based on Figure.1, one can write the coordinates of the centers $z_{j}(j=1,2,3)$ of the pendulum's rods in the forms: $z_{1}\left(a \sin \Omega t+\frac{\ell_{1}}{2} \sin \varphi_{1}, a \cos \Omega t+\frac{\ell_{1}}{2} \cos \varphi_{1}\right)$,

$z_{2}\left(a \sin \Omega t+\ell_{1} \sin \varphi_{1}+\frac{\ell_{2}}{2} \sin \varphi_{2}, a \cos \Omega t+\right.$

$\left.\ell_{1} \cos \varphi_{1}+\frac{\ell_{2}}{2} \cos \varphi_{2}\right)$,

$z_{3}\left(a \sin \Omega t+\ell_{1} \sin \varphi_{1}+\ell_{2} \sin \varphi_{2}+\frac{\ell_{3}}{2} \sin \varphi_{3}\right.$,

$\left.a \cos \Omega t+\ell_{1} \cos \varphi_{1}+\ell_{2} \cos \varphi_{2}+\frac{\ell_{3}}{2} \cos \varphi_{3}\right)$.

To gain more insight into the derivation of the governing equations of motion (GEOM), we are going to express the kinetic energy (KE) of the TP as the summation of the translation KE produced from the linear velocities $V_{c j}$ of the centers of mass and the rotation $\mathrm{KE}$ of each rod. Therefore, we can write easily the total $\mathrm{KE}$ in the form:

$T=\frac{1}{2} \sum_{j=1}^{3}\left[m_{j} V_{c j}^{2}+I_{j} \dot{\varphi}_{j}^{2}\right] ; I_{j}=\frac{m_{j} \ell_{j}^{2}}{12}(j=1,2,3)$

where $I_{j}$ represent moments of inertia of each rod. Referring to the above, the total kinetic and potential energies take the forms:

$$
\begin{aligned}
T & =\frac{1}{2} m_{1}\left[a^{2} \Omega^{2}+\frac{1}{4} \ell_{1}^{2} \dot{\varphi}_{1}^{2}+a \Omega \ell_{1} \cos \left(\Omega t-\varphi_{1}\right) \dot{\varphi}_{1}\right] \\
& +\frac{1}{2} m_{2}\left[a^{2} \Omega^{2}+\ell_{1}^{2} \dot{\varphi}_{1}^{2}+\frac{1}{4} \ell_{2}^{2} \dot{\varphi}_{2}^{2}+2 a \Omega \ell_{1} \dot{\varphi}_{1}\right. \\
& \times \cos \left(\Omega t-\varphi_{1}\right)+a \Omega \ell_{2} \cos \left(\Omega t-\varphi_{2}\right) \dot{\varphi}_{2}+\ell_{1} \ell_{2} \\
& \left.\times \cos \left(\varphi_{1}-\varphi_{2}\right) \dot{\varphi}_{1} \dot{\varphi}_{2}\right]+\frac{1}{2} m_{3}\left[a^{2} \Omega^{2}+\ell_{1}^{2} \dot{\varphi}_{1}^{2}+\ell_{2}^{2} \dot{\varphi}_{2}^{2}\right. \\
& +\frac{1}{4} \ell_{3}^{2} \dot{\varphi}_{3}^{2}+2 a \Omega \ell_{1} \cos \left(\Omega t-\varphi_{1}\right) \dot{\varphi}_{1}+2 a \Omega \ell_{2} \dot{\varphi}_{2} \\
& \times \cos \left(\Omega t-\varphi_{2}\right)+a \Omega \ell_{3} \cos \left(\Omega t-\varphi_{3}\right) \dot{\varphi}_{3}+2 \ell_{1} \ell_{2} \dot{\varphi}_{1} \dot{\varphi}_{2} \\
& \times \cos \left(\varphi_{1}-\varphi_{2}\right)+\ell_{1} \ell_{3} \cos \left(\varphi_{1}-\varphi_{3}\right) \dot{\varphi}_{1} \dot{\varphi}_{3}+\ell_{2} \ell_{3} \dot{\varphi}_{2} \dot{\varphi}_{3} \\
& \left.\times \cos \left(\varphi_{2}-\varphi_{3}\right)\right]+\frac{1}{24}\left[m_{1} \ell_{1}^{2} \dot{\varphi}_{1}^{2}+m_{2} \ell_{2}^{2} \dot{\varphi}_{2}^{2}+m_{3} \ell_{3}^{2} \dot{\varphi}_{3}^{2}\right], \\
V & =-m_{1} g\left(a \cos \Omega t+\frac{\ell_{1}}{2} \cos \varphi_{1}\right)-m_{2} g(a \cos \Omega t \\
& \left.+\ell_{1} \cos \varphi_{1}+\frac{\ell_{2}}{2} \cos \varphi_{2}\right)-m_{3} g(a \cos \Omega t \\
& \left.+\ell_{1} \cos \varphi_{1}+\ell_{2} \cos \varphi_{2}+\frac{\ell_{3}}{2} \cos \varphi_{3}\right) .
\end{aligned}
$$


By (4) and (5), the Lagrangian $L=T-V$ can be obtained easily, then using the following Lagrange's equations (Awrejcewicz, 2012) to obtain the GEOM as follows:

$\frac{d}{d t}\left(\frac{\partial L}{\partial \dot{\varphi}_{1}}\right)-\left(\frac{\partial L}{\partial \varphi_{1}}\right)=Q_{\varphi_{1}}$,

$\frac{d}{d t}\left(\frac{\partial L}{\partial \dot{\varphi}_{2}}\right)-\left(\frac{\partial L}{\partial \varphi_{2}}\right)=0$,

$\frac{d}{d t}\left(\frac{\partial L}{\partial \dot{\varphi}_{3}}\right)-\left(\frac{\partial L}{\partial \varphi_{3}}\right)=Q_{\varphi_{3}}$.

Here, $\varphi_{j}$ and $\dot{\varphi}_{j}(j=1,2,3)$ are the generalized coordinates and velocities respectively, while $Q_{\varphi_{1}}$ and $Q_{\varphi_{3}}$ are the generalized forces.

$$
\begin{aligned}
& Q_{\varphi_{1}}=M(t)=M_{1}(t) \cos \left(\Omega_{1} t\right), \\
& Q_{\varphi_{3}}=F(t)=F_{0} \cos \left(\Omega_{2} t\right),
\end{aligned}
$$

where, $F_{0}$ is the amplitude of the external force $F(t)$, while $\Omega_{1}$ and $\Omega_{2}$ denote the forcing frequencies of $M(t)$ and $F(t)$ respectively.

The final goal of this section is to obtain the GEOM in a dimensionless form. To achieve this goal we are going to consider the following forms of dimensionless coordinate, frequencies, parameters, and time.

$$
\begin{aligned}
& \varpi=\frac{\Omega}{w_{1}}, w_{j}^{2}=\frac{g}{\ell_{j}}(j=1,2,3), \omega_{2}^{2}=\frac{w_{2}^{2}}{w_{1}^{2}}, \omega_{3}^{2}=\frac{w_{3}^{2}}{w_{1}^{2}}, \\
& p_{1}=\frac{\Omega_{1}}{w_{1}}, p_{2}=\frac{\Omega_{2}}{w_{1}}, c_{1}=\frac{3 \ell_{2}\left(m_{2}+2 m_{3}\right)}{2 \ell_{1}\left(m_{1}+3 m_{2}+3 m_{3}\right)}, \\
& c_{2}=\frac{3 \ell_{3} m_{3}}{2 \ell_{1}\left(m_{1}+3 m_{2}+3 m_{3}\right)}, c_{3}=\frac{3\left(m_{1}+2 m_{2}+2 m_{3}\right)}{2\left(m_{1}+3 m_{2}+3 m_{3}\right)}, \\
& f_{1}=\frac{3 M_{1}(t)}{\ell_{1}^{2} w_{1}^{2}\left(m_{1}+3 m_{2}+3 m_{3}\right)}, b_{1}=\frac{3 \ell_{1}\left(m_{2}+2 m_{3}\right)}{2 \ell_{2}\left(m_{2}+3 m_{3}\right)}, \\
& b_{2}=\frac{3 \ell_{3} m_{3}}{2 \ell_{2}\left(m_{2}+3 m_{3}\right)}, b_{3}=\frac{3\left(m_{2}+2 m_{3}\right)}{2\left(m_{2}+3 m_{3}\right)}, s_{1}=\frac{3 \ell_{1}}{2 \ell_{3}}, \\
& s_{2}=\frac{3 \ell_{2}}{2 \ell_{3}}, f_{2}=\frac{3 F_{o}}{\ell_{3}^{2} w_{1}^{2} m_{3}}, \tau=w_{1} t .
\end{aligned}
$$

Substitution of (4), (5), (7), (8) into (6), yields the GEOM in the following dimensionless forms:

$$
\begin{aligned}
& \ddot{\varphi}_{1}+c_{1} \cos \left(\varphi_{1}-\varphi_{2}\right) \ddot{\varphi}_{2}+c_{2} \cos \left(\varphi_{1}-\varphi_{3}\right) \ddot{\varphi}_{3}+c_{1} \dot{\varphi}_{2}^{2} \\
& \times \sin \left(\varphi_{1}-\varphi_{2}\right)+c_{2} \sin \left(\varphi_{1}-\varphi_{3}\right) \dot{\varphi}_{3}^{2}-\frac{a}{\ell_{1}} \varpi^{2} c_{3} \\
& \times \sin \left(\varpi \tau-\varphi_{1}\right)+c_{3} \sin \varphi_{1}=f_{1} \cos \left(p_{1} \tau\right), \\
& \ddot{\varphi}_{2}+b_{1} \cos \left(\varphi_{1}-\varphi_{2}\right) \ddot{\varphi}_{1}+b_{2} \cos \left(\varphi_{2}-\varphi_{3}\right) \ddot{\varphi}_{3}-b_{1} \dot{\varphi}_{1}^{2} \\
& \times \sin \left(\varphi_{1}-\varphi_{2}\right)+b_{2} \sin \left(\varphi_{2}-\varphi_{3}\right) \dot{\varphi}_{3}^{2}-\frac{a}{\ell_{2}} \varpi^{2} b_{3} \\
& \times \sin \left(\varpi \tau-\varphi_{2}\right)+\omega_{2}{ }^{2} b_{3} \sin \varphi_{2}=0, \\
& \ddot{\varphi}_{3}+s_{1} \cos \left(\varphi_{1}-\varphi_{3}\right) \ddot{\varphi}_{1}+s_{2} \cos \left(\varphi_{2}-\varphi_{3}\right) \ddot{\varphi}_{2}-s_{1} \dot{\varphi}_{1}^{2} \\
& \sin \left(\varphi_{1}-\varphi_{3}\right)-s_{2} \sin \left(\varphi_{2}-\varphi_{3}\right) \dot{\varphi}_{2}^{2}-\frac{3}{2} \frac{a}{\ell_{3}} \varpi^{2} \\
& \times \sin \left(\varpi \tau-\varphi_{3}\right)+\frac{3}{2} \omega_{3}^{2} \sin \varphi_{3}=f_{2} \cos \left(p_{2} \tau\right) .
\end{aligned}
$$

Looking closely at the system of equations (9)-(11), we conclude that it is a nonlinear system of differential equations of second order in $\varphi_{j}$ which are functions of the dimensionless parameter $\tau=w_{1} t$.

\section{Methodology}

In this section, we are going to use MST to achieve the asymptotic solutions of the GEOM (9)-(11) and to obtain the resonance conditions (Rajasekar \& Sanjuan., 2016). Therefore, we approximate the trigonometric functions of $\varphi_{j}(j=1,2,3)$ these equations up to the third order. It must be noted that these approximations are valid in a tiny neighborhood of the static equilibrium position. Therefore equations (9)-(11) become 


$$
\begin{aligned}
& \ddot{\varphi}_{1}+\frac{c_{1}}{36}\left[\varphi_{1} \varphi_{2}\left(\varphi_{1}^{2}-6\right)\left(\varphi_{2}^{2}-6\right)+9\left(\varphi_{1}^{2}-2\right)\right. \\
& \left.\times\left(\varphi_{2}^{2}-2\right)\right] \ddot{\varphi}_{2}+\frac{c_{2}}{36}\left[\varphi_{1} \varphi_{3}\left(\varphi_{1}^{2}-6\right)\left(\varphi_{3}^{2}-6\right)\right. \\
& \left.+9\left(\varphi_{1}^{2}-2\right)\left(\varphi_{3}^{2}-2\right)\right] \ddot{\varphi}_{3}+\frac{c_{1}}{12}\left[\varphi_{1}\left(\varphi_{1}^{2}-6\right)\right. \\
& \left.\times\left(\varphi_{2}^{2}-2\right)-\varphi_{2}\left(\varphi_{1}^{2}-2\right)\left(\varphi_{2}^{2}-6\right)\right] \dot{\varphi}_{2}^{2}+\frac{c_{2}}{12}\left[\varphi_{1}\right. \\
& \left.\times\left(\varphi_{1}^{2}-6\right)\left(\varphi_{3}^{2}-2\right)-\varphi_{3}\left(\varphi_{1}^{2}-2\right)\left(\varphi_{3}^{2}-6\right)\right] \dot{\varphi}_{3}^{2} \\
& -\frac{a}{\ell_{1}} \varpi^{2} c_{3}\left[\sin (\varpi \tau)\left(1-\frac{\varphi_{1}^{2}}{2}\right)-\cos (\varpi \tau)\left(\varphi_{1}\right.\right. \\
& \left.\left.-\frac{\varphi_{1}^{3}}{6}\right)\right]+c_{3}\left(\varphi_{1}-\frac{\varphi_{1}^{3}}{6}\right)=f_{1} \cos \left(p_{1} \tau\right), \\
& \ddot{\varphi}_{2}+\frac{b_{1}}{36}\left[\varphi_{1} \varphi_{2}\left(\varphi_{1}^{2}-6\right)\left(\varphi_{2}^{2}-6\right)+9\left(\varphi_{1}^{2}-2\right)\right. \\
& \left.\times\left(\varphi_{2}^{2}-2\right)\right] \ddot{\varphi}_{1}+\frac{b_{2}}{36}\left[\varphi_{2} \varphi_{3}\left(\varphi_{2}^{2}-6\right)\left(\varphi_{3}^{2}-6\right)\right. \\
& \left.+9\left(\varphi_{2}^{2}-2\right)\left(\varphi_{3}^{2}-2\right)\right] \ddot{\varphi}_{3}-\frac{b_{1}}{12}\left[\varphi_{1}\left(\varphi_{1}^{2}-6\right)\right. \\
& \left.\times\left(\varphi_{2}^{2}-2\right)-\varphi_{2}\left(\varphi_{1}^{2}-2\right)\left(\varphi_{2}^{2}-6\right)\right] \dot{\varphi}_{1}^{2}+\frac{b_{2}}{12}\left[\varphi_{2}\right. \\
& \left.\times\left(\varphi_{2}^{2}-6\right)\left(\varphi_{3}^{2}-2\right)-\varphi_{3}\left(\varphi_{2}^{2}-2\right)\left(\varphi_{3}^{2}-6\right)\right] \dot{\varphi}_{3}^{2} \\
& -\frac{a}{\ell_{2}} \varpi^{2} b_{3}\left[\sin (\varpi \tau)\left(1-\frac{\varphi_{2}^{2}}{2}\right)-\cos (\varpi \tau)\right. \\
& \left.\times\left(\varphi_{2}-\frac{\varphi_{2}^{3}}{6}\right)\right]+\omega_{2}{ }^{2} b_{3}\left(\varphi_{2}-\frac{\varphi_{2}^{3}}{6}\right)=0, \\
& \ddot{\varphi}_{3}+\frac{s_{1}}{36}\left[\varphi_{1} \varphi_{3}\left(\varphi_{1}^{2}-6\right)\left(\varphi_{3}^{2}-6\right)+9\left(\varphi_{1}^{2}-2\right)\right. \\
& \left.\times\left(\varphi_{3}^{2}-2\right)\right] \ddot{\varphi}_{1}+\frac{s_{2}}{36}\left[\varphi_{2} \varphi_{3}\left(\varphi_{2}^{2}-6\right)\left(\varphi_{3}^{2}-6\right)\right. \\
& \left.+9\left(\varphi_{2}^{2}-2\right)\left(\varphi_{3}^{2}-2\right)\right] \ddot{\varphi}_{2}-\frac{s_{1}}{12}\left[\varphi_{1}\left(\varphi_{1}^{2}-6\right)\right. \\
& \left.\times\left(\varphi_{3}^{2}-2\right)-\varphi_{3}\left(\varphi_{1}^{2}-2\right)\left(\varphi_{3}^{2}-6\right)\right] \dot{\varphi}_{1}^{2}-\frac{s_{2}}{12}\left[\varphi_{2}\right. \\
& \left.\times\left(\varphi_{2}^{2}-6\right)\left(\varphi_{3}^{2}-2\right)-\varphi_{3}\left(\varphi_{2}^{2}-2\right)\left(\varphi_{3}^{2}-6\right)\right] \dot{\varphi}_{2}^{2} \\
& -\frac{3}{2} \frac{a}{\ell_{3}} \varpi^{2}\left[\sin (\varpi \tau)\left(1-\frac{\varphi_{3}^{2}}{2}\right)-\cos (\varpi \tau)\right. \\
& \left.\times\left(\varphi_{3}-\frac{\varphi_{3}^{3}}{6}\right)\right]+\frac{3}{2} \omega_{3}^{2}\left(\varphi_{3}-\frac{\varphi_{3}^{3}}{6}\right)=f_{2} \cos \left(p_{2} \tau\right) .
\end{aligned}
$$

Now, we are going to introduce a small parameter $0<\varepsilon<<1$ in the previous equations (12)-(14). Therefore, we express the amplitude of oscillations in terms of $\varepsilon$ as follow:

$$
\begin{aligned}
& \varphi_{1}(\tau)=\varepsilon \phi(\tau ; \varepsilon), \quad \varphi_{2}(\tau)=\varepsilon \psi(\tau ; \varepsilon), \\
& \varphi_{3}(\tau)=\varepsilon \chi(\tau ; \varepsilon),
\end{aligned}
$$

According to MST procedure, we seek the second-order (three terms) expansions for $\phi, \psi$ and $\chi$ in powers $\varepsilon$ in the form:

$$
\begin{aligned}
& \phi=\sum_{k=0}^{2} \varepsilon^{k} \phi_{k+1}\left(\tau_{0}, \tau_{1}, \tau_{2}\right)+O\left(\varepsilon^{3}\right), \\
& \psi=\sum_{k=0}^{2} \varepsilon^{k} \psi_{k+1}\left(\tau_{0}, \tau_{1}, \tau_{2}\right)+O\left(\varepsilon^{3}\right), \\
& \chi=\sum_{k=0}^{2} \varepsilon^{k} \chi_{k+1}\left(\tau_{0}, \tau_{1}, \tau_{2}\right)+O\left(\varepsilon^{3}\right),
\end{aligned}
$$

where $\tau_{n}=\varepsilon^{n} \tau(n=0,1,2)$ represent different time scales.

To determine $\phi, \psi$ and $\chi$ as functions $\tau_{n}$, we change $\tau$ to $\tau_{n}$ according to the following chain rule:

$\frac{d}{d \tau}=\frac{\partial}{\partial \tau_{0}}+\varepsilon \frac{\partial}{\partial \tau_{1}}+\varepsilon^{2} \frac{\partial}{\partial \tau_{2}}$

$\frac{d^{2}}{d \tau^{2}}=\frac{\partial^{2}}{\partial \tau_{0}^{2}}+2 \varepsilon \frac{\partial^{2}}{\partial \tau_{0} \partial \tau_{1}}+\varepsilon^{2}\left(\frac{\partial^{2}}{\partial \tau_{1}^{2}}+2 \frac{\partial^{2}}{\partial \tau_{0} \partial \tau_{2}}\right)+O\left(\varepsilon^{3}\right)$.

Here, terms of $O\left(\varepsilon^{3}\right)$ and higher are neglected due to their smallness. Considering that:

$$
\begin{aligned}
& c_{j}=\varepsilon^{2} \tilde{c}_{j}, \quad b_{j}=\varepsilon^{2} \tilde{b}_{j}, \quad s_{j}=\varepsilon^{2} \tilde{s}_{j}, \quad c_{3}=\tilde{c}_{3}, \\
& b_{3}=\tilde{b}_{3}, \quad a=\varepsilon^{2} \tilde{a}, \quad f_{j}=\varepsilon^{3} \tilde{f}_{j}, \quad(j=1,2)
\end{aligned}
$$

in which the parameters $\tilde{c}_{j}, \tilde{b}_{j}, \tilde{s}_{j}, \tilde{c}_{3}, \tilde{b}_{3}, \tilde{a}$ and $\tilde{f}_{j}$ are of order 1 .

Substituting (15)-(18) into (12)-(14) and equating coefficients of equal powers of $\varepsilon, \varepsilon^{2}$ and $\varepsilon^{3}$ on both sides, to obtain:

Order of $\varepsilon^{1}$

$$
\begin{aligned}
& \frac{\partial^{2} \phi_{1}}{\partial \tau_{0}^{2}}+\tilde{c}_{3} \phi_{1}=0, \\
& \frac{\partial^{2} \psi_{1}}{\partial \tau_{0}^{2}}+\tilde{b}_{3} \omega_{2}^{2} \psi_{1}=0,
\end{aligned}
$$


$\frac{\partial^{2} \chi_{1}}{\partial \tau_{0}^{2}}+\frac{3}{2} \omega_{3}^{2} \chi_{1}=0$

Order of $\varepsilon^{2}$

$\left.\frac{\partial^{2} \phi_{2}}{\partial \tau_{0}^{2}}+\tilde{c}_{3} \phi_{2}=-2 \frac{\partial^{2} \phi_{1}}{\partial \tau_{0} \partial \tau_{1}}+\frac{\tilde{a} \tilde{c}_{3}}{\ell_{1}} \varpi^{2} \sin \left(\varpi \tau_{0}\right), \quad\right) 22($
$\frac{\partial^{2} \psi_{2}}{\partial \tau_{0}^{2}}+\tilde{b}_{3} \omega_{2}^{2} \psi_{2}=-2 \frac{\partial^{2} \psi_{1}}{\partial \tau_{0} \tau_{1}}+\frac{\tilde{a} \tilde{b}_{3}}{\ell_{2}} \varpi^{2} \sin \left(\varpi \tau_{0}\right),(23)$
$\frac{\partial^{2} \chi_{2}}{\partial \tau_{0}^{2}}+\frac{3}{2} \omega_{3}^{2} \chi_{2}=-2 \frac{\partial^{2} \chi_{1}}{\partial \tau_{0} \tau_{1}}+\frac{3}{2} \frac{\tilde{a}}{\ell_{3}} \varpi^{2} \sin \left(\varpi \tau_{0}\right)$,

Order of $\varepsilon^{3}$

$$
\begin{aligned}
& \frac{\partial^{2} \phi_{3}}{\partial \tau_{0}^{2}}+\tilde{c}_{3} \phi_{3}=\tilde{f_{1}} \cos \left(p_{1} \tau_{0}\right)-\frac{\partial^{2} \phi_{1}}{\partial \tau_{1}^{2}}-2\left(\frac{\partial^{2} \phi_{1}}{\partial \tau_{0} \partial \tau_{2}}\right. \\
& \left.+\frac{\partial^{2} \phi_{2}}{\partial \tau_{0} \partial \tau_{1}}\right)-\tilde{c}_{1} \frac{\partial^{2} \psi_{1}}{\partial \tau_{0}^{2}}-\tilde{c}_{2} \frac{\partial^{2} \chi_{1}}{\partial \tau_{0}^{2}} \\
& +\frac{\tilde{c_{3}}}{6} \phi_{1}^{3}-\frac{\tilde{a} \tilde{c}_{3}}{\ell_{1}} \varpi^{2} \cos \left(\varpi \tau_{0}\right) \phi_{1}, \\
& \frac{\partial^{2} \psi_{3}}{\partial \tau_{0}^{2}}+\tilde{b}_{3} \omega_{2}^{2} \psi_{3}=-\frac{\partial^{2} \psi_{1}}{\partial \tau_{1}^{2}}-2\left(\frac{\partial^{2} \psi_{1}}{\partial \tau_{0} \partial \tau_{2}}+\frac{\partial^{2} \psi_{2}}{\partial \tau_{0} \partial \tau_{1}}\right) \\
& -\tilde{b_{1}} \frac{\partial^{2} \phi_{1}}{\partial \tau_{0}^{2}}-\tilde{b_{2}} \frac{\partial^{2} \chi_{1}}{\partial \tau_{0}^{2}}+\frac{\tilde{b_{3}} \omega_{2}^{2}}{6} \psi_{1}^{3} \\
& -\frac{\tilde{a} \tilde{b}_{3}}{\ell_{2}} \varpi^{2} \cos \left(\varpi \tau_{0}\right) \psi_{1} \\
& \frac{\partial^{2} \chi_{3}}{\partial \tau_{0}^{2}}+\frac{3}{2} \omega_{3}^{2} \chi_{3}=\tilde{f_{2}} \cos \left(p_{2} \tau_{0}\right)-\frac{\partial^{2} \chi_{1}}{\partial \tau_{1}^{2}}-2\left(\frac{\partial^{2} \chi_{1}}{\partial \tau_{0} \partial \tau_{2}}\right. \\
& \left.+\frac{\partial^{2} \chi_{2}}{\partial \tau_{0} \partial \tau_{1}}\right)-\tilde{s}_{1} \frac{\partial^{2} \phi_{1}}{\partial \tau_{0}^{2}}-\tilde{s}_{2} \frac{\partial^{2} \psi_{1}}{\partial \tau_{0}^{2}}+\frac{\omega_{3}^{2}}{4} \chi_{1}^{3} \\
& -\frac{3}{2} \frac{\tilde{a}}{\ell_{3}} \varpi^{2} \cos \left(\varpi \tau_{0}\right) \chi_{1} .
\end{aligned}
$$

An inspection of the previous system of equations (19)-(27) reveals that we have three groups of partial differential equations (19)(21), (22)-(24), and (25)-(27), in which it can be solved subsequently. Therefore, we start with the general solutions of equations (19)(21) which take the forms:

$$
\begin{aligned}
& \phi_{1}=\mathrm{A}_{1} e^{i \sqrt{c_{3}} \tau_{0}}+\overline{\mathrm{A}}_{1} e^{-i \sqrt{c_{3}} \tau_{0}}, \\
& \psi_{1}=\mathrm{A}_{2} e^{i \sqrt{\bar{b}_{3}} \omega_{2} \tau_{0}}+\overline{\mathrm{A}}_{2} e^{-i \sqrt{b_{3}} \omega_{2} \tau_{0}}, \\
& \chi_{1}=\mathrm{A}_{3} e^{i \sqrt{\frac{3}{2} \omega_{3} \tau_{0}}}+\overline{\mathrm{A}}_{3} e^{-i \sqrt{\frac{3}{2}} \omega_{3} \tau_{0}},
\end{aligned}
$$

where, $\quad i=\sqrt{-1} \quad$ and $\quad \mathrm{A}_{j}(j=1,2,3)$ are unknown complex functions of slow time scales $\tau_{1}$ and $\tau_{2}$, while $\overline{\mathrm{A}}_{j}$ indicates to its complex conjugates.

Substituting (28)-(30) into (22)-(24) and eliminating terms that produce secular terms to obtain uniform asymptotic solutions. Then, the conditions for eliminating these terms are:

$\frac{\partial \mathrm{A}_{1}}{\partial \tau_{1}}=0, \quad \frac{\partial \mathrm{A}_{2}}{\partial \tau_{1}}=0, \quad \frac{\partial \mathrm{A}_{3}}{\partial \tau_{1}}=0$.

Therefore, we can write the second-order solutions in the forms:

$$
\begin{aligned}
& \phi_{2}=\frac{i \tilde{a} \tilde{c}_{3} \varpi^{2}}{2 \ell_{1}\left(\varpi^{2}-\tilde{c}_{3}\right)} e^{i \varpi \tau_{0}}+C C, \\
& \psi_{2}=\frac{i \tilde{a} \tilde{b}_{3} \varpi^{2}}{2 \ell_{2}\left(\varpi^{2}-\tilde{b}_{3} \omega_{2}^{2}\right)} e^{i \varpi \tau_{0}}+C C, \\
& \chi_{2}=\frac{3 i \tilde{a} \varpi^{2}}{2 \ell_{3}\left(2 \varpi^{2}-3 \omega_{3}^{2}\right)} e^{i \varpi \tau_{0}}+C C,
\end{aligned}
$$

where $C C$ are the complex conjugates of the preceding terms.

By the above, cancelling of secular terms demands that; the functions $\mathrm{A}_{j}(j=1,2,3)$ depend on $\tau_{2}$ only.

Substituting (28)-(31) and (32)-(34) into (25)(27), then cancelling terms that produce secular terms to obtain the third-order approximations. Elimination of these terms required:

$$
\begin{aligned}
& 2 i \sqrt{\tilde{c}_{3}} \frac{\partial \mathrm{A}_{1}}{\partial \tau_{2}}-\frac{\tilde{c_{3}}}{2} \mathrm{~A}_{1}^{2} \overline{\mathrm{A}}_{1}=0, \\
& 2 i \sqrt{\tilde{b}_{3}} \omega_{2} \frac{\partial \mathrm{A}_{2}}{\partial \tau_{2}}-\frac{\tilde{b_{3}}}{2} \omega_{2}^{2} \mathrm{~A}_{2}^{2} \overline{\mathrm{A}}_{2}=0, \\
& 2 i \sqrt{\frac{3}{2}} \omega_{3} \frac{\partial \mathrm{A}_{3}}{\partial \tau_{2}}-\frac{3}{4} \omega_{3}^{2} \mathrm{~A}_{3}^{2} \overline{\mathrm{A}}_{3}=0 .
\end{aligned}
$$


Thus, the solutions $\phi_{3}, \psi_{3}$ and $\chi_{3}$ have the forms:

$$
\begin{aligned}
& \phi_{3}=\frac{\tilde{f_{1}} e^{i p_{1} \tau_{0}}}{2\left(\tilde{c_{3}}-p_{1}^{2}\right)}+\frac{\tilde{c_{1}} \tilde{b}_{3} \omega_{2}^{2} \mathrm{~A}_{2} e^{i \tau_{0} \omega_{2} \sqrt{b_{3}}}}{\left(\tilde{c_{3}}-\tilde{b_{3}} \omega_{2}^{2}\right)}-\frac{\mathrm{A}_{1}^{3} e^{3 i \tau_{0} \sqrt{c_{3}}}}{48} \\
& +\frac{3 \tilde{c}_{2} \omega_{3}^{2} \mathrm{~A}_{3} e^{\sqrt{\frac{3}{2}} \tau_{0} \omega_{3}}}{\left(2 \tilde{c_{3}}-3 \omega_{3}^{2}\right)}+\frac{\tilde{a} \tilde{c}_{3} \varpi^{2} \mathrm{~A}_{1} e^{i \tau_{0}\left(\varpi+\sqrt{c_{3}}\right)}}{2 \ell_{1}\left(\varpi^{2}+2 \varpi \sqrt{\tilde{c}_{3}}\right)} \\
& +\frac{\tilde{a} \tilde{c}_{3} \varpi^{2} \overline{\mathrm{A}}_{1} e^{i \tau_{0}\left(\varpi-\sqrt{c_{3}}\right)}}{2 \ell_{1}\left(\varpi^{2}-2 \varpi \sqrt{\tilde{c}_{3}}\right)}+C C, \\
& \psi_{3}=\frac{\tilde{c}_{3} \tilde{b}_{1} \mathrm{~A}_{1} e^{i \tau_{0} \sqrt{c_{3}}}}{\left(\tilde{b}_{3} \omega_{2}^{2}-\tilde{c}_{3}\right)}+\frac{3 \tilde{b}_{2} \omega_{3}^{2} \mathrm{~A}_{3} e^{\sqrt{\frac{3}{2}} \tau_{0} \omega_{3}}}{\left(2 \tilde{b}_{3} \omega_{2}^{2}-3 \omega_{3}^{2}\right)} \\
& -\frac{\mathrm{A}_{2}^{3} e^{3 i \tau_{0} \omega_{2} \sqrt{b_{3}}}}{48}+\frac{\tilde{a} \tilde{b}_{3} \varpi^{2} \mathrm{~A}_{2} e^{i \tau_{0}\left(\varpi+\omega_{2} \sqrt{\tilde{b}_{3}}\right)}}{2 \ell_{2}\left(\varpi^{2}+2 \varpi \omega_{2} \sqrt{\tilde{b}_{3}}\right)} \\
& +\frac{\tilde{a} \tilde{b}_{3} \varpi^{2} \overline{\mathrm{A}}_{2} e^{i \tau_{0}\left(\varpi-\omega_{2} \sqrt{\tilde{b}_{3}}\right)}}{2 \ell_{2}\left(\varpi^{2}-2 \varpi \omega_{2} \sqrt{\tilde{b}_{3}}\right)}+C C, \\
& \chi_{3}=\frac{\tilde{f_{2}} e^{i p_{2} \tau_{0}}}{2\left(\frac{3}{2} \omega_{3}^{2}-p_{2}^{2}\right)}+\frac{\tilde{c_{3}} \tilde{s_{1}} \mathrm{~A}_{1} e^{i \tau_{0} \sqrt{c_{3}}}}{\left(\frac{3}{2} \omega_{3}^{2}-\tilde{c}_{3}\right)}-\frac{\mathrm{A}_{3}^{3} e^{3 \sqrt{\frac{3}{2}} i \tau_{0} \omega_{3}}}{48} \\
& +\frac{\tilde{s_{2}} \tilde{b_{3}} \omega_{2}^{2} \mathrm{~A}_{2} e^{i \tau_{0} \omega_{2} \sqrt{b_{3}}}}{\left(\frac{3}{2} \omega_{3}^{2}-\tilde{b_{3}} \omega_{2}^{2}\right)}+\frac{3 \tilde{a} \varpi^{2} \mathrm{~A}_{3} e^{i \tau_{0}\left(\varpi+\sqrt{\frac{3}{2}} \omega_{3}\right)}}{4 \ell_{3}\left(\varpi^{2}+\sqrt{6} \varpi \omega_{3}\right)} \\
& +\frac{3 \tilde{a} \varpi^{2} \overline{\mathrm{A}}_{3} e^{i \tau_{0}\left(\varpi-\sqrt{\left.\frac{3}{2} \omega_{3}\right)}\right.}}{4 \ell_{3}\left(\varpi^{2}-\sqrt{6} \varpi \omega_{3}\right)}+C C .
\end{aligned}
$$

The complex functions $\mathrm{A}_{j}(j=1,2,3)$ can be determined from conditions (31) and (53)-(37) system with the help of the following initial conditions:

$$
\begin{array}{lll}
\varphi_{1}(0)=z_{01}, & \varphi_{2}(0)=z_{03}, & \varphi_{3}(0)=z_{05}, \\
\dot{\varphi}_{1}(0)=z_{02}, & \dot{\varphi}_{2}(0)=z_{04}, & \dot{\varphi}_{3}(0)=z_{06},
\end{array}
$$

where, $z_{01}, z_{02}, \ldots, z_{06}$ are known initial quantities.

\section{Resonance cases}

In this section, we shed light on the classification of resonance cases. It is well known that resonance occurs when the denominators of the second and third approximations tend to zero. Therefore, many resonance cases can be detected from equations (32)-(34) and (38)-(40) in which can be classified into:

(i) Primary external occurs at

$$
p_{1} \approx \sqrt{\widetilde{c_{3}}}, \quad p_{2} \approx \sqrt{\frac{3}{2}} \omega_{3},
$$

(ii) Internal occurs at

$$
\sqrt{\tilde{b}_{3}} \omega_{2} \approx \sqrt{\tilde{c}_{3}}, \sqrt{\frac{3}{2}} \omega_{3} \approx \sqrt{\tilde{c}_{3}}, \sqrt{\frac{3}{2}} \omega_{3} \approx \sqrt{\tilde{b}_{3}} \omega_{2},
$$

(iii) Natural occurs at

$$
\begin{aligned}
& \varpi \approx \sqrt{\tilde{c_{3}}}, \varpi \approx \sqrt{\tilde{b_{3}}} \omega_{2}, \varpi \approx \sqrt{\frac{3}{2}} \omega_{3}, \\
& \varpi \approx 2 \sqrt{\tilde{c_{3}}}, \varpi \approx 2 \sqrt{\tilde{b_{3}}} \omega_{2}, \\
& \varpi \approx \sqrt{6} \omega_{3} .
\end{aligned}
$$

It is worthwhile to notice that, if any one of the resonance cases is satisfied, we can foretell that, the behaviour of the studied model will be very intricate. Moreover, the above approaches are valid if the vibrations have values outside of the resonance ones.

Now, we examine a combination of primary external and internal resonance in which they are simultaneously occurring.

Therefore, we consider:

$$
p_{1} \approx \sqrt{\tilde{c_{3}}}, p_{2} \approx \sqrt{\frac{3}{2}} \omega_{3} \text { and } \sqrt{\frac{3}{2}} \omega_{3}=\sqrt{\tilde{b_{3}}} \omega_{2} \text {, which }
$$

characterize the closeness of $p_{1}, p_{2}$ and $\sqrt{\frac{3}{2}} \omega_{3}$ to $\sqrt{\tilde{c}_{3}}, \sqrt{\frac{3}{2}} \omega_{3}$ and $\sqrt{\tilde{b}_{3}} \omega_{2}$ respectively. Therefore, we introduce what so-called detuning parameters $\sigma_{j}(j=1,2,3)$ as follows:

$$
\begin{aligned}
& p_{1}=\sqrt{\tilde{c}_{3}}+\sigma_{1}, \quad \sqrt{\frac{3}{2}} \omega_{3}=\sqrt{\tilde{b}_{3}} \omega_{2}+\sigma_{2}, \\
& p_{2}=\sqrt{\frac{3}{2}} \omega_{3}+\sigma_{3} .
\end{aligned}
$$

These parameters are considered as a gauge of 
the distance from the rigorous resonance. Consequently, we express about them in terms of $\varepsilon$ as:

$\sigma_{j}=\varepsilon^{2} \tilde{\sigma}_{j} ; \quad(j=1,2,3)$

Substituting (42) and (43) into (12)-(14), and taking into account the elimination of secular terms, we obtain the solvability conditions in the forms:

-According to the second-order approximation $\frac{\partial \mathrm{A}_{1}}{\partial \tau_{1}}=0, \quad \frac{\partial \mathrm{A}_{2}}{\partial \tau_{1}}=0, \quad \frac{\partial \mathrm{A}_{3}}{\partial \tau_{1}}=0$.

-According to the third-order approximation

$\tilde{f_{1}} e^{i \tau_{2} \tilde{\sigma}_{1}}-4 i \sqrt{\tilde{c}_{3}} \frac{\partial \mathrm{A}_{1}}{\partial \tau_{2}}+\tilde{c_{3}} \mathrm{~A}_{1}^{2} \overline{\mathrm{A}}_{1}=0$,

$4 i \sqrt{\tilde{b}_{3}} \omega_{2} \frac{\partial \mathrm{A}_{2}}{\partial \tau_{2}}-\tilde{b_{3}} \omega_{2}^{2} \mathrm{~A}_{2}^{2} \overline{\mathrm{A}}_{2}-3 \tilde{b_{2}} \omega_{3}^{2} \mathrm{~A}_{3} e^{i \tau_{2} \tilde{\sigma}_{2}}=0$,

$2 \tilde{f_{2}} e^{i \tau_{2} \tilde{\sigma}_{3}}-4 \sqrt{6} i \omega_{3} \frac{\partial \mathrm{A}_{3}}{\partial \tau_{2}}+3 \omega_{3}^{2} \mathrm{~A}_{3}^{2} \overline{\mathrm{A}}_{3}$

$+4 \tilde{s}_{2} \tilde{b}_{3} \omega_{2}^{2} \mathrm{~A}_{2} e^{-i \tau_{2} \tilde{\sigma}_{2}}=0$.

The unknown function $\mathrm{A}_{j}(j=1,2,3)$ can be obtained from (44) and (45) in which $\mathrm{A}_{j}$ depend only on $\tau_{2}$ Expressing $\mathrm{A}_{j}$ in polar forms as:

$\mathrm{A}_{j}=\frac{1}{2} \tilde{h}_{j}\left(\tau_{2}\right) e^{i \tilde{\gamma}_{j}\left(\tau_{2}\right)}, h_{j}=\varepsilon \tilde{h}_{j} \quad(j=1,2,3)$

where $\tilde{h}_{j}$ and $\tilde{\gamma}_{j}$ are real functions and refer to the amplitudes and phases of the solutions $\phi, \psi$ and $\chi$.

Because $\mathrm{A}_{j}$ are dependent functions of a variable $\tau_{2}$ only, the operator of the first-order derivative can be written in the form:

$\frac{d \mathrm{~A}_{j}}{d \tau}=\varepsilon^{2} \frac{\partial \mathrm{A}_{j}}{\partial \tau_{2}} ; \quad(j=1,2,3)$.

Taking into consideration the previous formula (47), equations (45) will be converted into ordinary differential equations (ODE). Introducing the following modified phases to transform the system of ODE into autonomous ones:

$\theta_{1}\left(\tau_{2}\right)=\tau_{2} \tilde{\sigma}_{1}-\gamma_{1}\left(\tau_{2}\right)$

$\theta_{2}\left(\tau_{2}\right)=\gamma_{2}\left(\tau_{2}\right)-\tau_{2} \tilde{\sigma}_{2}-\gamma_{3}\left(\tau_{2}\right)$,

$\theta_{3}\left(\tau_{2}\right)=\tau_{2} \tilde{\sigma}_{3}-\gamma_{3}\left(\tau_{2}\right)$,

Substituting (46)-(48) into (45) and separating real and imaginary parts to obtain the following desired autonomous system:

$$
\begin{aligned}
& 16 \sqrt{c_{3}} h_{1} \frac{d \theta_{1}}{d \tau}=8 f_{1} \cos \theta_{1}+16 \sqrt{c_{3}} h_{1} \sigma_{1}+c_{3} h_{1}^{3}, \\
& 2 \sqrt{c_{3}} \frac{d h_{1}}{d \tau}=f_{1} \sin \theta_{1}, \\
& 16 \sqrt{b_{3}} \omega_{2} h_{2} \frac{d \theta_{2}}{d \tau}=\omega_{2} h_{2}\left[16 \sqrt{b_{3}}\left(\frac{d \theta_{3}}{d \tau}-\sigma_{2}-\sigma_{3}\right)\right. \\
& \left.\quad-b_{3} \omega_{2} h_{2}^{2}\right]-12 b_{2} h_{3} \omega_{3}^{2} \cos \theta_{2}, \\
& 4 \sqrt{b_{3}} \omega_{2} \frac{d h_{2}}{d \tau}=-3 b_{2} h_{3} \omega_{3}^{2} \sin \theta_{2}, \\
& \sqrt{6} \omega_{3} h_{3} \frac{d \theta_{3}}{d \tau}=\omega_{3} h_{3}\left(\sqrt{6} \sigma_{3}+\frac{3}{16} \omega_{3} h_{3}^{2}\right) \\
& \quad+s_{2} h_{2} b_{3} \omega_{2}^{2} \cos \theta_{2}+f_{2} \cos \theta_{3}, \\
& \sqrt{6} \omega_{3} \frac{d h_{3}}{d \tau}=s_{2} h_{2} b_{3} \omega_{2}^{2} \sin \theta_{2}+f_{2} \sin \theta_{3} .
\end{aligned}
$$

This system describes the modulation equations of both amplitudes and phases $h_{j}$ and $\theta_{j}(j=1,2,3)$ respectively, which consists of a system of six ODE from first order when three resonances occur simultaneously. On the other side, the solutions of this system are plotted in Figures.2-7 when we consider the following data:

$$
\begin{aligned}
& m_{1}=(4,10,15) \mathrm{kg}, \quad m_{2}=(5,9,12) \mathrm{kg}, \\
& m_{3}=(3,8,12) \mathrm{kg}, \quad \ell_{1}=(0.6,1,1.5) \mathrm{m}, \\
& \ell_{2}=(0.5,0.9,1.5) \mathrm{m}, \ell_{3}=(0.4,0.8,1.2) \mathrm{m}, \\
& \Omega_{1}=w_{1} p_{1}, \quad \Omega_{2}=w_{2} p_{2}, \quad \Omega=0.2 \mathrm{rad} . \mathrm{s}^{-1}, \\
& \sigma_{1}=0.2, \quad \sigma_{3}=0.0205, \quad \sigma_{2}=\sqrt{\frac{3}{2}} \omega_{3}-\sqrt{b_{3}} \omega_{2} \\
& F_{0}=0.001 \mathrm{~N}, \quad M_{1}=0.02 \mathrm{~N} . \mathrm{m} .
\end{aligned}
$$

The objective of the calculations presented in these figures is to study the variation of 
different values of masses $m_{j}$ and lengths of pendulum arms $\ell_{j}$, that correspond to $\mathrm{w}_{i}(\mathrm{j}=1,2,3)$. Inspection of Figure. 2 show that the behaviour of the waves describe the amplitude.

$h_{1}$ has a periodic form when $m_{j}$ and $\ell_{j}$ are changed. It is remarked that the number of oscillations does not change with the change of masses, on the contrary, the amplitude of the waves decreases when $m_{j}$ increases, see parts $(a),(b)$ and $(c)$ of Figure.2.

Moreover, the variation of the values $\ell_{1}$ has a good impact on $h_{1}$, see a part $(d)$, of the same figure while different values of $\ell_{2}$ and $\ell_{3}$ have the same effect as seen in parts $(e)$ and $(f)$ due to the second equation in (49).
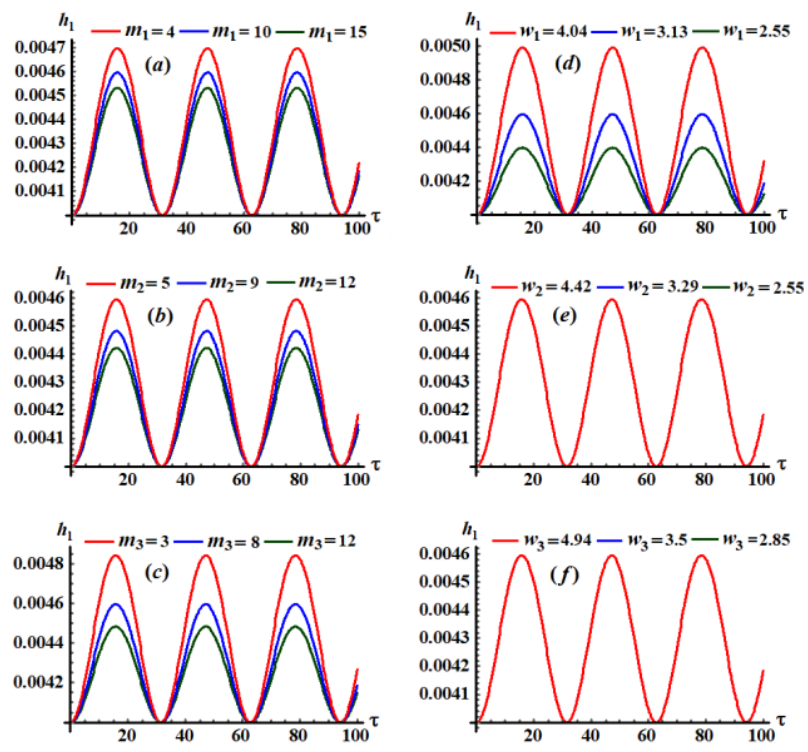

Fig.2. Represents modulation of the amplitude $h_{1}$ versus time $\tau$ at $(a)$ $m_{1}=(4,10,15) \mathrm{kg}, \quad$ (b) $\quad m_{2}=(5,9,12) \mathrm{kg}, \quad$ (c) $m_{3}=(3,8,12) \mathrm{kg},(\boldsymbol{d}) w_{1}=(4.04,3.13,2.55) \mathrm{s}^{-1}, \quad(\boldsymbol{e})$ $w_{2}=(4.42,3.29,2.55) \mathrm{s}^{-1},(\boldsymbol{f}) w_{3}=(4.94,3.5,2.85) \mathrm{s}^{-1}$.
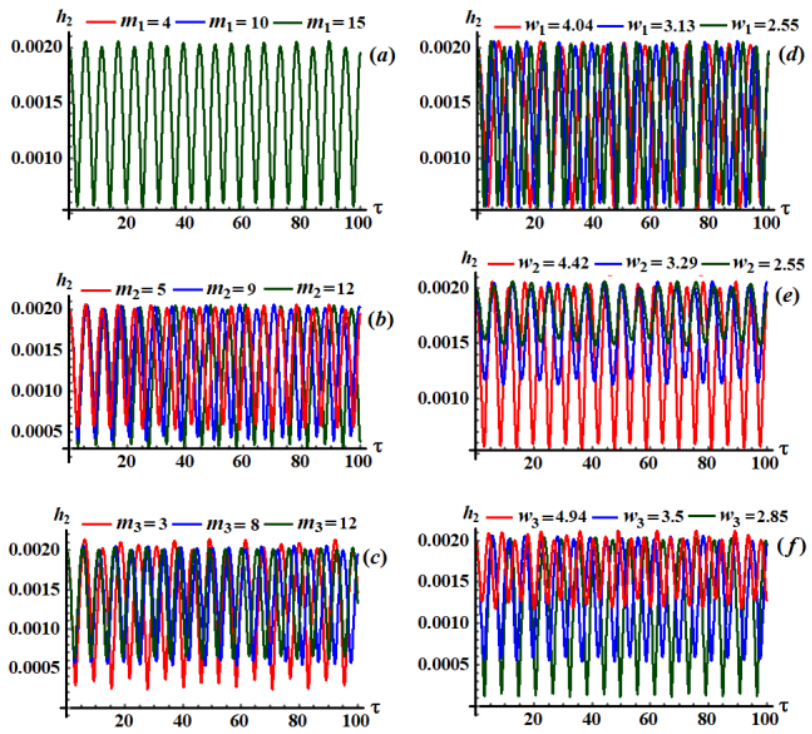

Fig.3. Illustrates variation of the amplitude $h_{2} \quad$ via $\tau$ at $($ a $) m_{1}=(4,10,15) \mathrm{kg}$, (b) $m_{2}=(5,9,12) \mathrm{kg}, \quad$ (c) $m_{3}=(3,8,12) \mathrm{kg}, \quad$ (d) $w_{1}=(4.04,3.13,2.55) s^{-1},(\boldsymbol{e}) w_{2}=(4.42,3.29,2.55) \mathrm{s}^{-1}$, (f) $w_{3}=(4.94,3.5,2.85) s^{-1}$.

Looking closely at Figures.3 and 4, we find that $h_{2}$ and $h_{3}$ are strongly influenced by the change of both masses $m_{j}$ and lengths $\ell_{j}$ due to the fourth and sixth equations in (49) in which the number of oscillations increases and the amplitudes of the waves decrease to some extent.

It is worthwhile to notice Figures.5 and 7 that, the modified phases $\theta_{1}$ and $\theta_{3}$ increase gradually when time goes on, in which this increment is repeated many times due to the plane motion and the nature of these modified phases. 

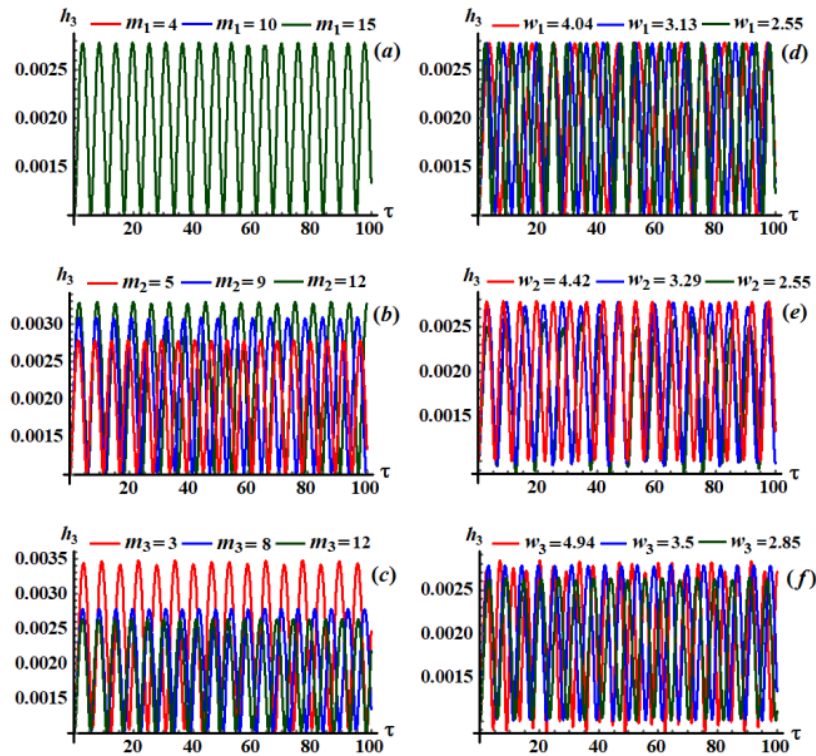

Fig.4. Describes variation of $h_{3}$ via $\tau$ at $(a)$ $m_{1}=(4,10,15) \mathrm{kg}, \quad$ (b) $\quad m_{2}=(5,9,12) \mathrm{kg}, \quad(\boldsymbol{c})$ $m_{3}=(3,8,12) \mathrm{kg}, \quad(\boldsymbol{d}) w_{1}=(4.04,3.13,2.55) \mathrm{s}^{-1},(\boldsymbol{e})$ $w_{2}=(4.42,3.29,2.55) s^{-1},(f) w_{3}=(4.94,3.5,2.85) s^{-1}$.
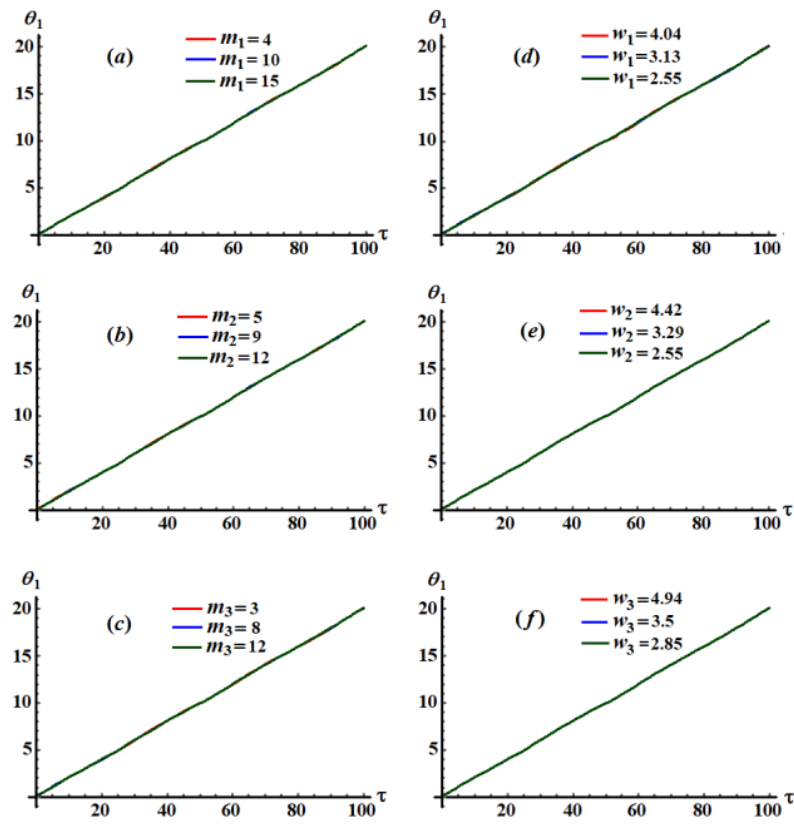

Fig.5. Represents modulation of the modified phase $\theta_{1}$ versus time $\tau$ at $(a)$ $m_{1}=(4,10,15) \mathrm{kg}, \quad(\boldsymbol{b}) \quad m_{2}=(5,9,12) \mathrm{kg}, \quad(\boldsymbol{c})$ $m_{3}=(3,8,12) \mathrm{kg}, \quad(\boldsymbol{d}) \quad w_{1}=(4.04,3.13,2.55) \mathrm{s}^{-1}, \quad(\boldsymbol{e})$ $w_{2}=(4.42,3.29,2.55) \mathrm{s}^{-1},(\boldsymbol{f}) w_{3}=(4.94,3.5,2.85) \mathrm{s}^{-1}$.
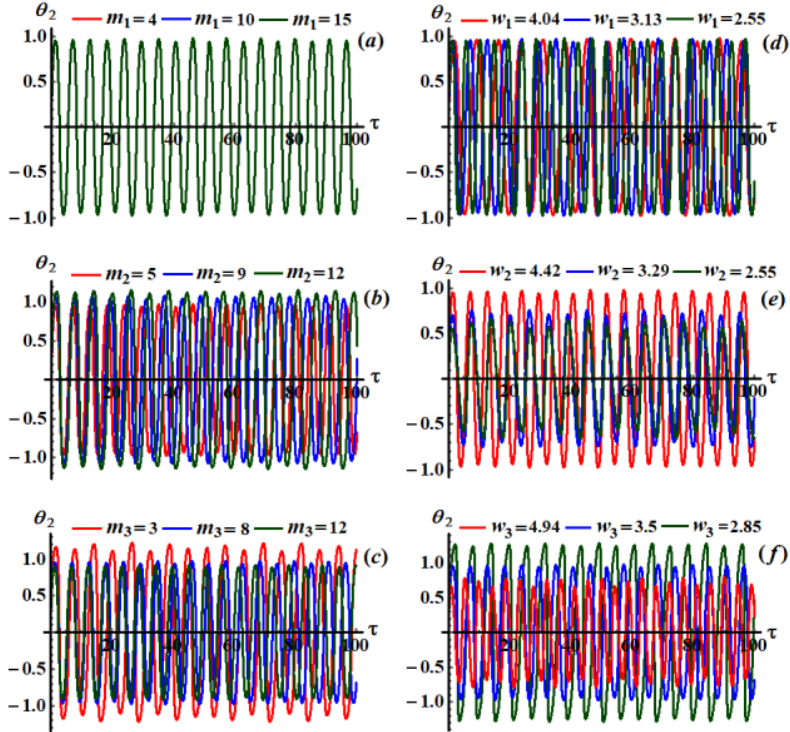

Fig.6. Examines the influence of different values of mass $m_{1}, m_{2}, m_{3}$ and $w_{1}, w_{2}, w_{3}$ on the modified phase $\theta_{2}$.
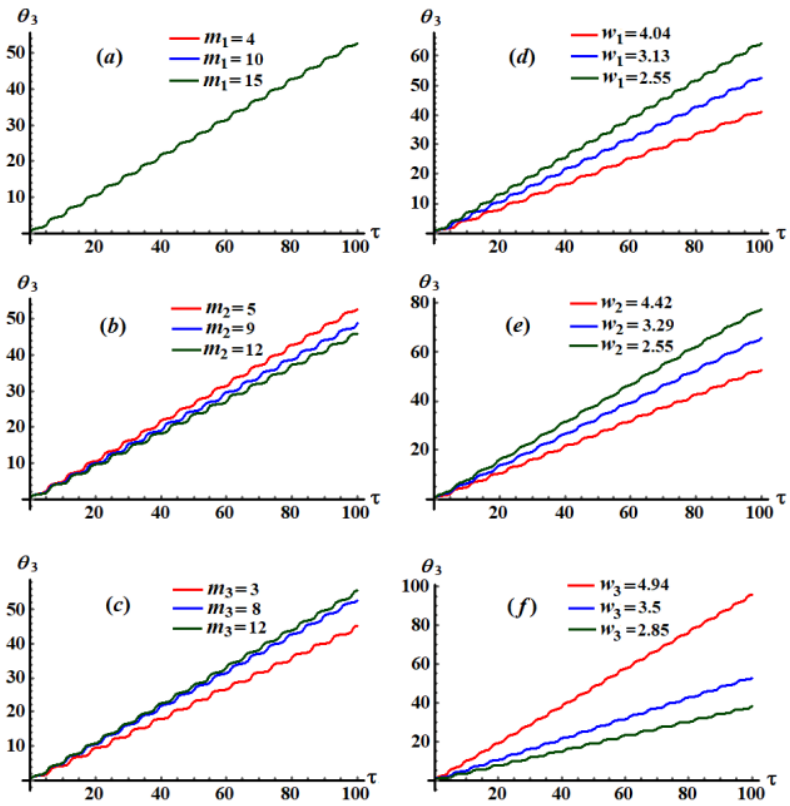

Fig.7. Examines the influence of different values of mass $m_{1}, m_{2}, m_{3}$ and $w_{1}, w_{2}, w_{3}$ on the modified phase $\theta_{3}$. 

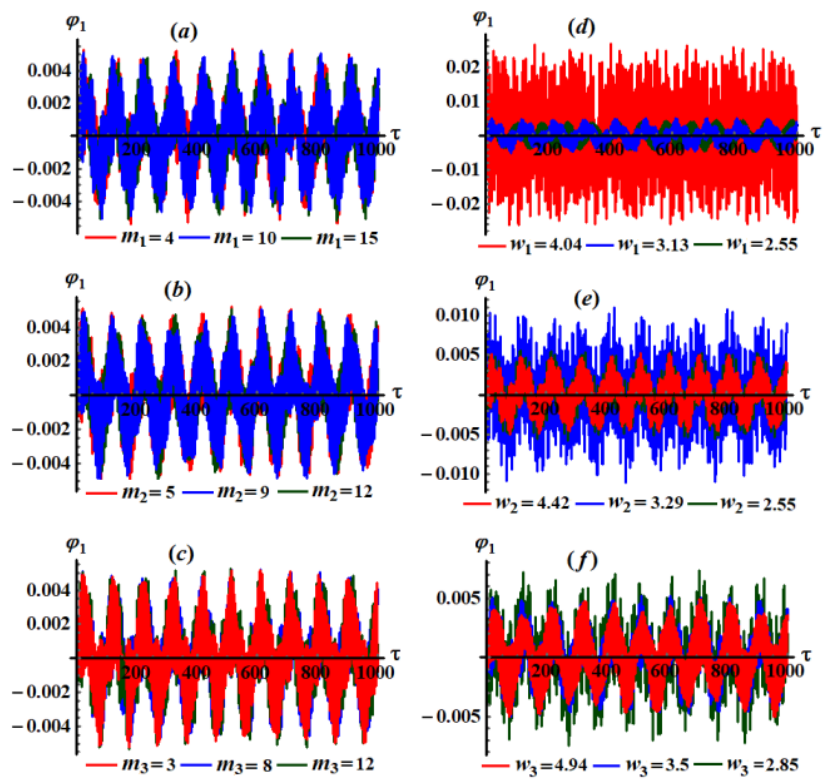

Fig.8. Reveals the effects of variation of mass $m_{1}, m_{2}, m_{3}$ and $w_{1}, w_{2}, w_{3}$ on the $\operatorname{solution} \varphi_{1}$ •
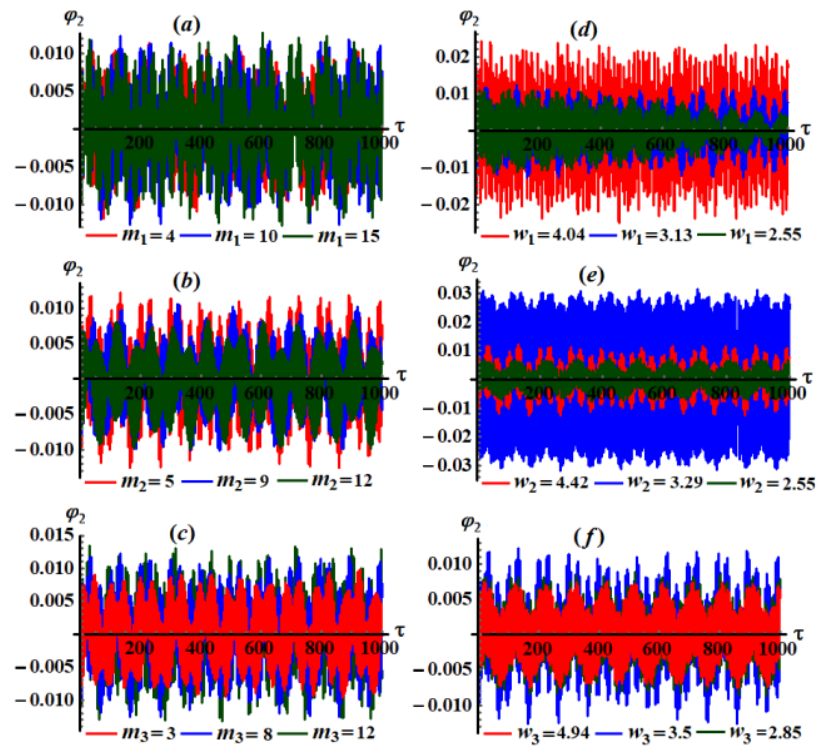

Fig.9. Reveals the effects of variation of mass $m_{1}, m_{2}, m_{3}$ and $w_{1}, w_{2}, w_{3}$ on the solution $\varphi_{2}$ •

It is obvious that $\theta_{1}$ has the same effect with the change of $m_{j}$ and $\ell_{j}$ values as indicated in Figure.5, while $m_{j}$ and $\ell_{j}$ have a good influence on the manner of $\theta_{3}$ as mentioned in Figure.7. On the other side, it should be noticed from Figure. 6 that, the modified phase $\theta_{2}$ oscillates rapidly with short-wavelength when $m_{j}$ and $\ell_{j}$ increases.

The principle aim from Figures.8-10 is to investigate the time history of the solutions $\varphi_{j}(j=1,2,3)$ when $m_{j}$ and $\ell_{j}$ have different values as mentioned before. All of these solutions have periodic oscillations during the whole time which indicates that the dynamical motion of the considered model is stable and free of chaos.
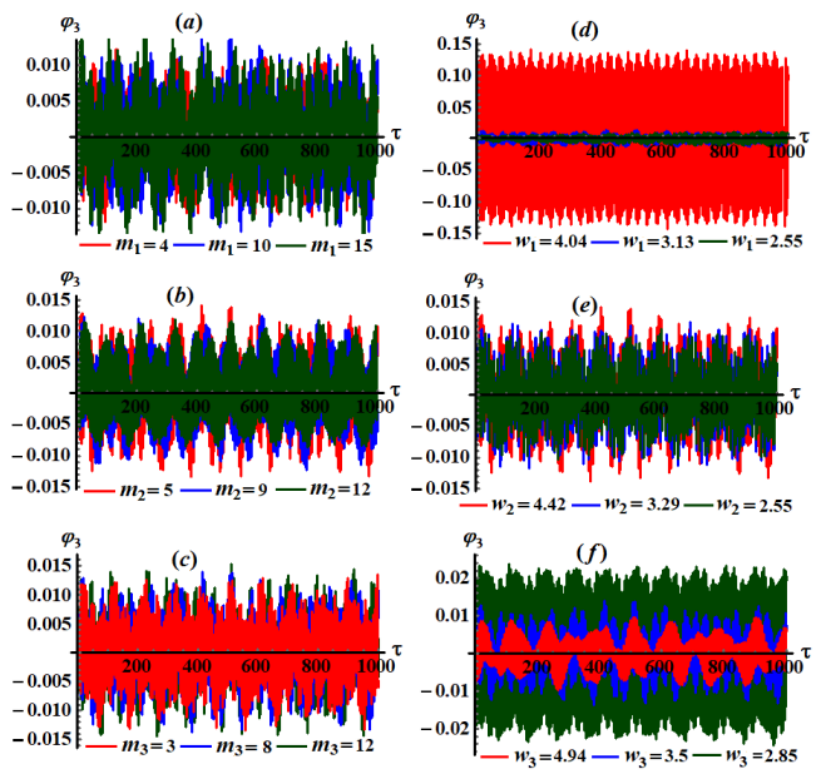

Fig.10.: Shows a variation of the time history of the solution $\varphi_{3}$ when $m_{1}, m_{2}, m_{3}$ and $w_{1}, w_{2}, w_{3}$ have different values.

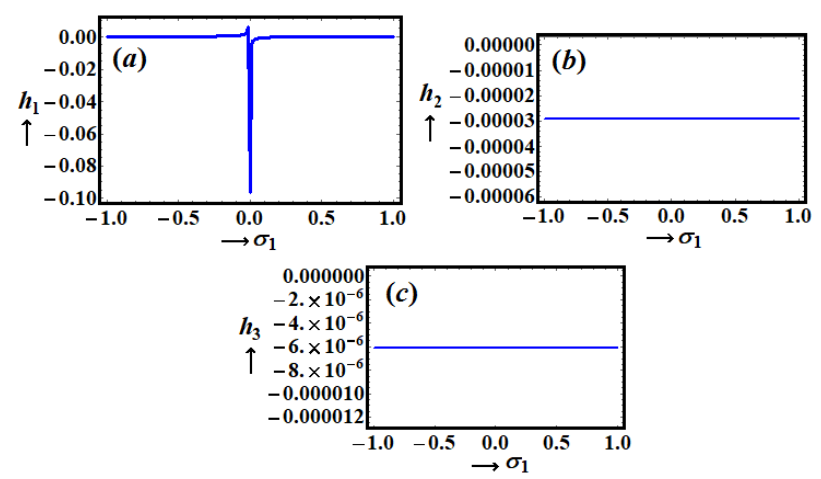

Fig.11. Shows resonance curves of amplitudes $h_{j}(j=1,2,3)$ as functions of $\sigma_{1}$ at $\sigma_{2}=-0.12816, \sigma_{3}=0.0205$. 


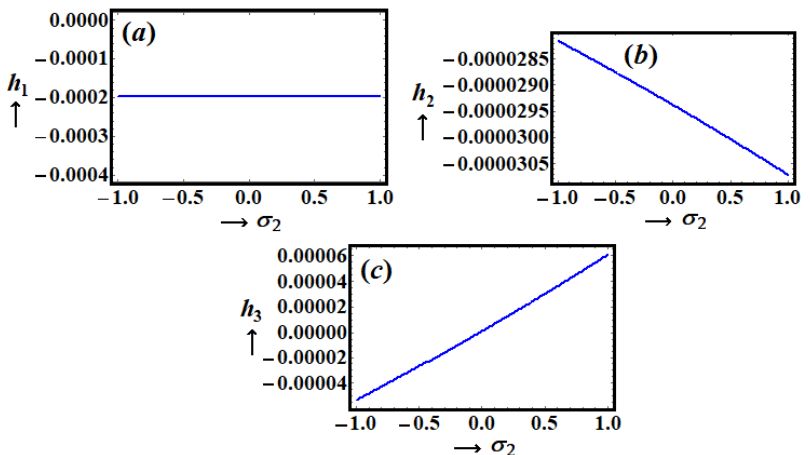

Fig.12. Shows resonance curves of amplitudes $h_{j}(j=1,2,3)$ as functions of $\sigma_{2}$ at $\sigma_{1}=0.2, \sigma_{3}=0.0205$.
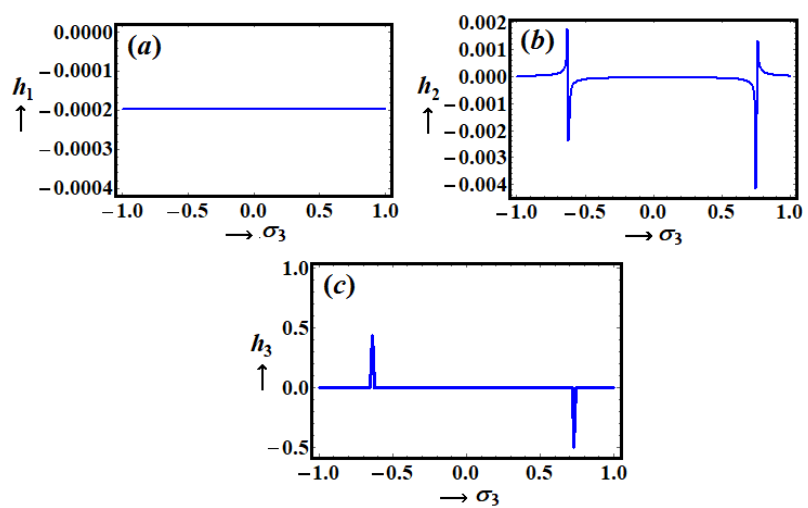

Fig.13. Presents resonance curves of amplitudes $h_{j}(j=1,2,3)$ as functions of $\sigma_{3}$ at $\sigma_{1}=0.2, \sigma_{2}=-0.12816$.

\section{Steady-state solutions}

The task of this section is to investigate the steady-state solutions of our model that correspond to the zero derivative of amplitudes $h_{j}$ and modified phases $\theta_{j}(j=1,2,3)$. Therefore, we consider

$\frac{d h_{j}}{d t}=\frac{d \theta_{j}}{d t}=0 \quad(j=1,2,3)$

Making use of (49) and (50), we obtain the following algebraic equations:

$$
\begin{aligned}
& 8 f_{1} \cos \theta_{1}+16 \sqrt{c_{3}} h_{1} \sigma_{1}+c_{3} h_{1}^{3}=0, \\
& f_{1} \sin \theta_{1}=0, \\
& 16 \omega_{2} h_{2} \sqrt{b_{3}}\left(\sigma_{2}+\sigma_{3}\right)+b_{3} \omega_{2}^{2} h_{2}^{3} \\
& +12 b_{2} h_{3} \omega_{3}^{2} \cos \theta_{2}=0, \\
& b_{2} h_{3} \omega_{3}^{2} \sin \theta_{2}=0, \\
& \omega_{3} h_{3}\left(\sqrt{6} \sigma_{3}+\frac{3}{16} \omega_{3} h_{3}^{2}\right)+s_{2} h_{2} b_{3} \omega_{2}^{2} \\
& \times \cos \theta_{2}+f_{2} \cos \theta_{3}=0, \\
& s_{2} h_{2} b_{3} \omega_{2}^{2} \sin \theta_{2}+f_{2} \sin \theta_{3}=0 .
\end{aligned}
$$

Eliminating the modified phases $\theta_{j}$ from equations (51), yield

$$
\begin{aligned}
& 8 f_{1}+16 \sqrt{c_{3}} h_{1} \sigma_{1}+c_{3} h_{1}^{3}=0, \\
& 16 \omega_{2} h_{2} \sqrt{b_{3}}\left(\sigma_{2}+\sigma_{3}\right)+b_{3} \omega_{2}^{2} h_{2}^{3}+12 b_{2} h_{3} \omega_{3}^{2}=0, \\
& \omega_{3} h_{3}\left(\sqrt{6} \sigma_{3}+\frac{3}{16} \omega_{3} h_{3}^{2}\right)+s_{2} h_{2} b_{3} \omega_{2}^{2}+f_{2}=0 .
\end{aligned}
$$

These equations represent the frequency response functions clarified by the detuning parameters in terms of the amplitudes. Therefore, resonance curves are represented graphically in Figures.11-13.

\section{Nonlinear analysis}

This section investigates the stability of a TP motion as a dynamical model utilizing a nonlinear stability analysis approach. The motion of this model is considered under the action of an external harmonic force $F(t)$. Criteria stability is achieved besides the computer emulation of the non-linear evolution equations system. Some parameters like $\ell_{j}, m_{j}$ as well as $\sigma_{j}(j=1,2,3)$ play a destabilizing role in the stabilization process.

To indicate the properties of the nonlinear amplitude for system (49) and discuss its stability, the following transformations are introduced: 
$\mathrm{A}_{1}=\frac{1}{2}\left(\tilde{U}_{1}\left(\tau_{2}\right)+i \tilde{V}_{1}\left(\tau_{2}\right)\right) e^{i \tilde{\sigma}_{1} \tau_{2}}$

$\mathrm{A}_{2}=\frac{1}{2}\left(\tilde{U}_{2}\left(\tau_{2}\right)+i \tilde{V}_{2}\left(\tau_{2}\right)\right) e^{i\left(\tilde{\sigma}_{2}+\tilde{\sigma}_{3}\right) \tau_{2}}$

$\mathrm{A}_{3}=\frac{1}{2}\left(\tilde{U}_{3}\left(\tau_{2}\right)+i \tilde{V}_{3}\left(\tau_{2}\right)\right) e^{i \tilde{\sigma}_{3} \tau_{2}}$,

$U_{j}=\varepsilon \tilde{U}_{j}, V_{j}=\varepsilon \tilde{V}_{j}, \quad \sigma_{j}=\varepsilon^{2} \tilde{\sigma}_{j}(j=1,2,3)$.

Therefore, separating the real and imaginary parts to get:

$$
\begin{aligned}
& 16 \sqrt{c_{3}}\left(\frac{d V_{1}}{d \tau}+\sigma_{1} U_{1}\right)+c_{3} U_{1}\left(U_{1}^{2}+V_{1}^{2}\right)+8 f_{1}=0 \\
& 16 \sqrt{c_{3}}\left(\frac{d U_{1}}{d \tau}-\sigma_{1} V_{1}\right)-c_{3} V_{1}\left(U_{1}^{2}+V_{1}^{2}\right)=0 \\
& 16 \sqrt{b_{3}} \omega_{2}\left[\frac{d V_{2}}{d \tau}+\left(\sigma_{2}+\sigma_{3}\right) U_{2}\right]+b_{3} \omega_{2}^{2} U_{2} \\
& \times\left(U_{2}^{2}+V_{2}^{2}\right)+12 b_{2} U_{3} \omega_{3}^{2}=0 \\
& 16 \sqrt{b_{3}} \omega_{2}\left[\frac{d U_{2}}{d \tau}-\left(\sigma_{2}+\sigma_{3}\right) V_{2}\right]-b_{3} \omega_{2}^{2} V_{2} \\
& \times\left(U_{2}^{2}+V_{2}^{2}\right)-12 b_{2} V_{3} \omega_{3}^{2}=0 \\
& 8 \sqrt{6} \omega_{3}\left(\frac{d V_{3}}{d \tau}+\sigma_{3} U_{3}\right)+3 \omega_{3}^{2} U_{3}\left(U_{3}^{2}+V_{3}^{2}\right) \\
& +8\left(s_{2} b_{3} \omega_{2}^{2} U_{2}+f_{2}\right)=0 \\
& 8 \sqrt{6} \omega_{3}\left(\frac{d U_{3}}{d \tau}-\sigma_{3} V_{3}\right)-3 \omega_{3}^{2} V_{3}\left(U_{3}^{2}+V_{3}^{2}\right) \\
& -8 s_{2} b_{3} \omega_{2}^{2} V_{2}=0
\end{aligned}
$$

The modified amplitudes were subsequently justified in whole time interval for different parametric values and the properties of the amplitudes are plotted in phase plane curves as indicated in Figures.14-22 in which the following data for the fixed physical values are considered:

$$
\begin{aligned}
& m_{1}=10 \mathrm{~kg}, m_{2}=5 \mathrm{~kg}, m_{3}=8 \mathrm{~kg}, M_{1}=0.02 \mathrm{~N} . \mathrm{m}, \\
& F_{0}=0.001 \mathrm{~N}, \quad \ell_{1}=1 \mathrm{~m}, \ell_{2}=0.5 \mathrm{~m}, \ell_{3}=0.8 \mathrm{~m}, \\
& \Omega=0.2 \mathrm{rad} . \mathrm{s}^{-1}, \Omega_{1}=w_{1} p_{1}, \Omega_{2}=w_{2} p_{2}, \varepsilon=0.005, \\
& \sigma_{1}=0.2, \sigma_{3}=0.0205, \sigma_{2}=\sqrt{\frac{3}{2}} \omega_{3}-\sqrt{b_{3}} \omega_{2} .
\end{aligned}
$$

Figure.14 reveals the projection of the path of the modulation equation on the phase plane $U_{1} V_{1}$ and the time history of the modified phases $U_{1}$ and $V_{1}$ with time $\tau$ when $\ell_{1}=1.5 m, w_{1}=2.55 s^{-1}$. Its shape can be circular. Also, the development of the amplitudes $U_{2}, V_{2}$ and $U_{3}, V_{2}$ versus time are presented in Figures.15 and 16 for the same parameters considered in Figure.14 of the studied system. Moreover, Figures.17-19 reveal the phase portrait $U_{j} V_{j}(j=1,2,3)$ planes and the development of the amplitudes $U_{j} V_{j}$ and against time under the impact of the variation $\ell_{2}$. Within this context, Figures.2022 examine the effect of the variation of the parameters $\ell_{3}$.

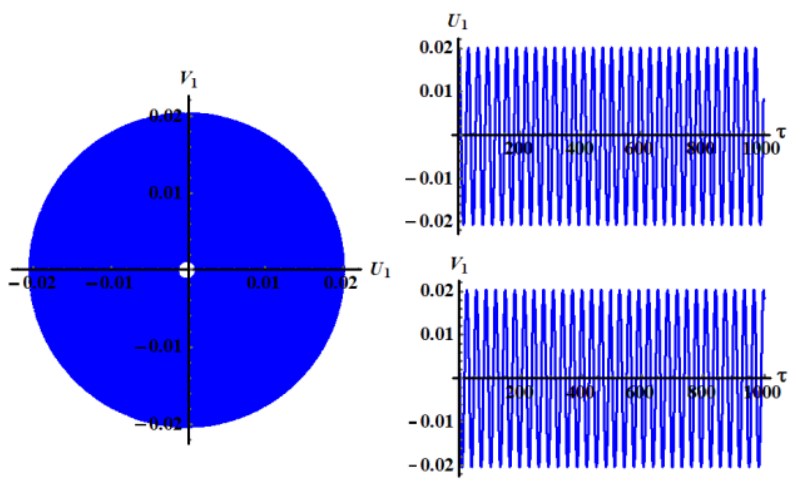

Fig.14. The projection of the path of the modulation equation on $U_{1} V_{1}$ the phase plane and the modified amplitudes versus time $\tau$ when $\ell_{1}=1.5 \mathrm{~m}, w_{1}=2.55 \mathrm{~s}^{-1}$.
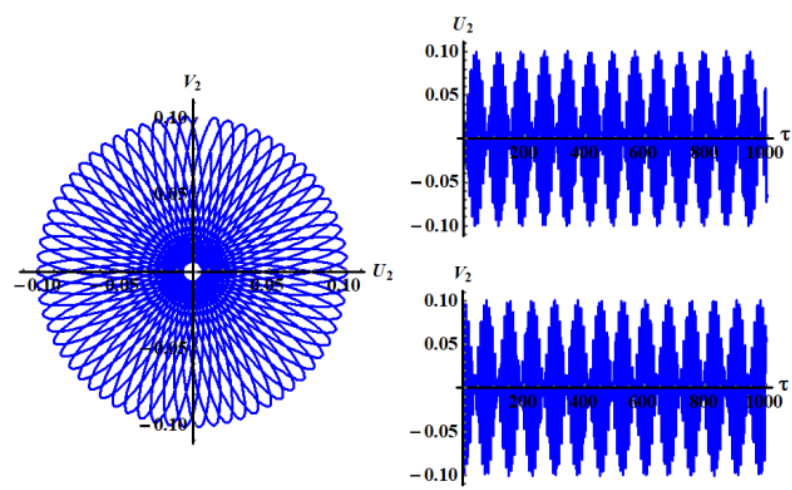

Fig.15. Represents $U_{2} V_{2}$ phase plane and the history of the amplitudes $U_{2}$ and $V_{2}$ at $\ell_{1}=1.5 m, w_{1}=2.55 \mathrm{~s}^{-1}$. 

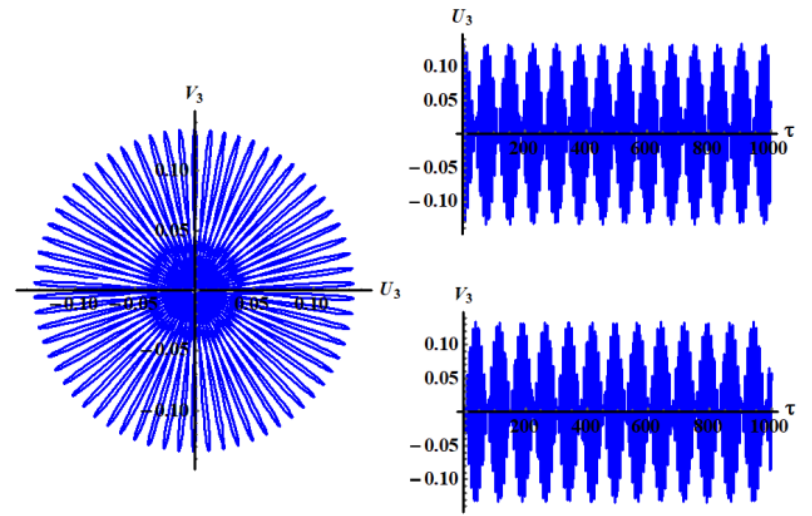

Fig.16. Illustrates $U_{3} V_{3}$ phase plane and the time history of the amplitudes $U_{3}$ and $V_{3}$ at $\ell_{1}=1.5 m, w_{1}=2.55 \mathrm{~s}^{-1}$.
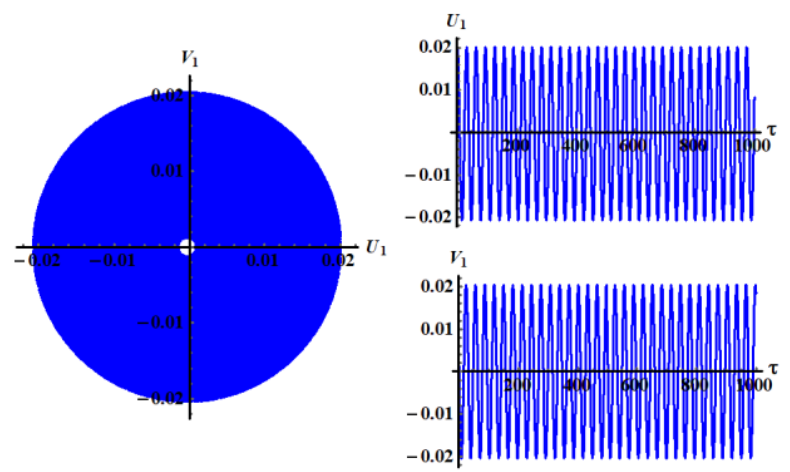

Fig.17. Describes $U_{1} V_{1}$ phase plane and the variation of the amplitudes $U_{1}$ and $V_{1}$ with $\tau$ when $\ell_{2}=1.5 m, w_{2}=2.55 \mathrm{~s}^{-1}$.
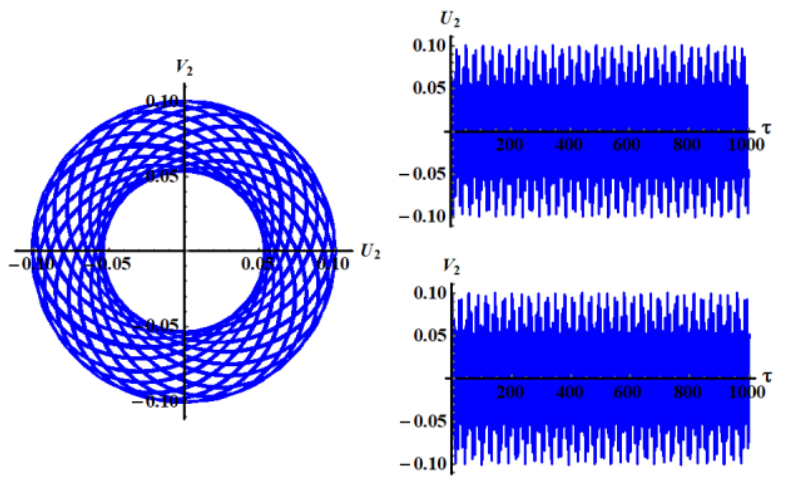

Fig.18. Shows $U_{2} V_{2}$ phase plane diagram and the variation of the amplitudes $U_{2}$ and $V_{2}$ with $\tau$ when $\ell_{2}=1.5 m, w_{2}=2.55 \mathrm{~s}^{-1}$.
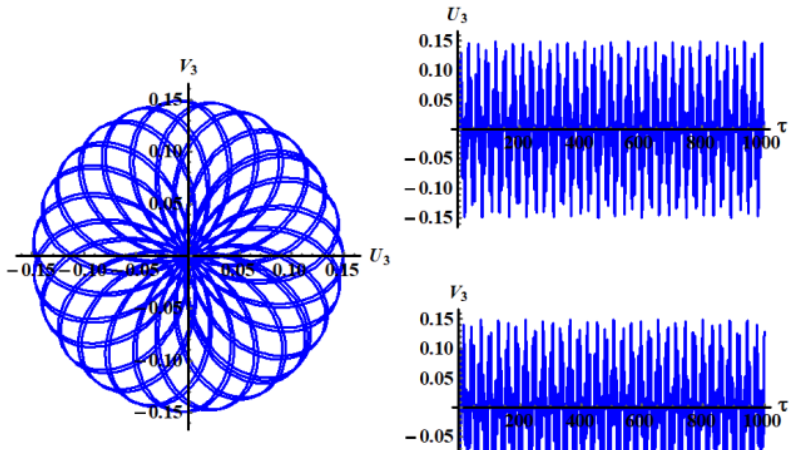

Fig.19. The projection of the path of the modulation equation on $U_{3} V_{3}$ the phase plane and the modified amplitudes via time $\tau$ at $\ell_{2}=1.5 m, w_{2}=2.55 \mathrm{~s}^{-1}$.
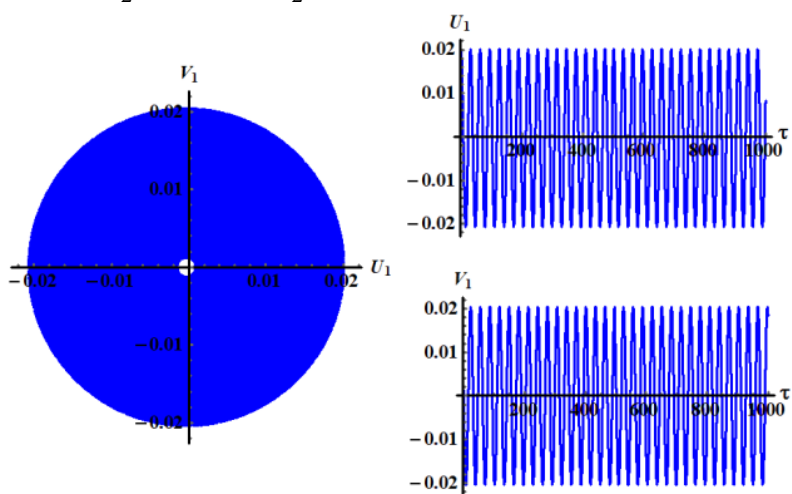

Fig.20. The projection of the path of the modulation equation on $U_{1} V_{1}$ the phase plane and the modified amplitudes via time $\tau$ at $\ell_{3}=1.2 \mathrm{~m}, w_{3}=2.857 \mathrm{~s}^{-1}$.
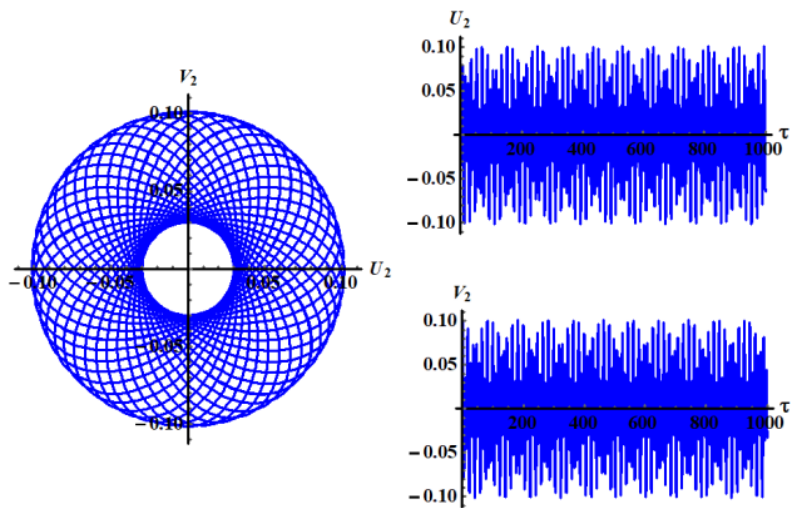

Fig.21. Shows $U_{2} V_{2}$ phase plane diagram and the variation of the amplitudes $U_{2}$ and $V_{2}$ with $\tau$ when $\ell_{3}=1.2 \mathrm{~m}, w_{3}=2.857 \mathrm{~s}^{-1}$. 


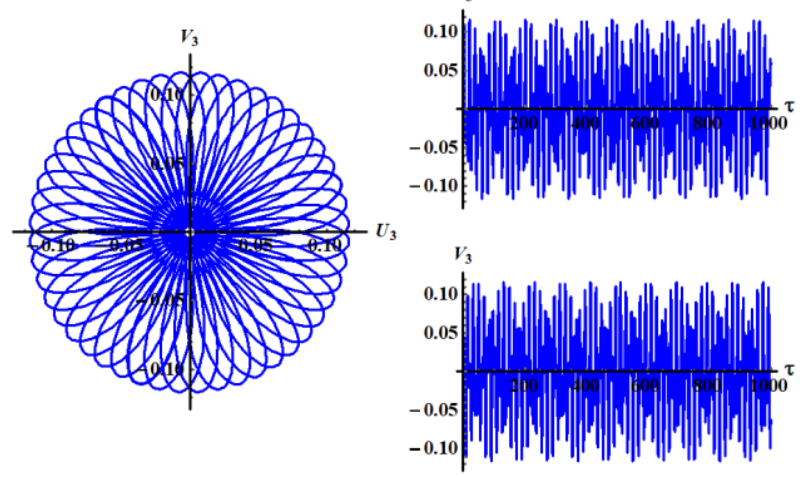

Fig.22. Describes $U_{3} V_{3}$ phase plane diagram and time history of the amplitudes $U_{3}$ and $V_{3}$ when $\ell_{3}=1.2 m, w_{3}=2.857 \mathrm{~s}^{-1}$.
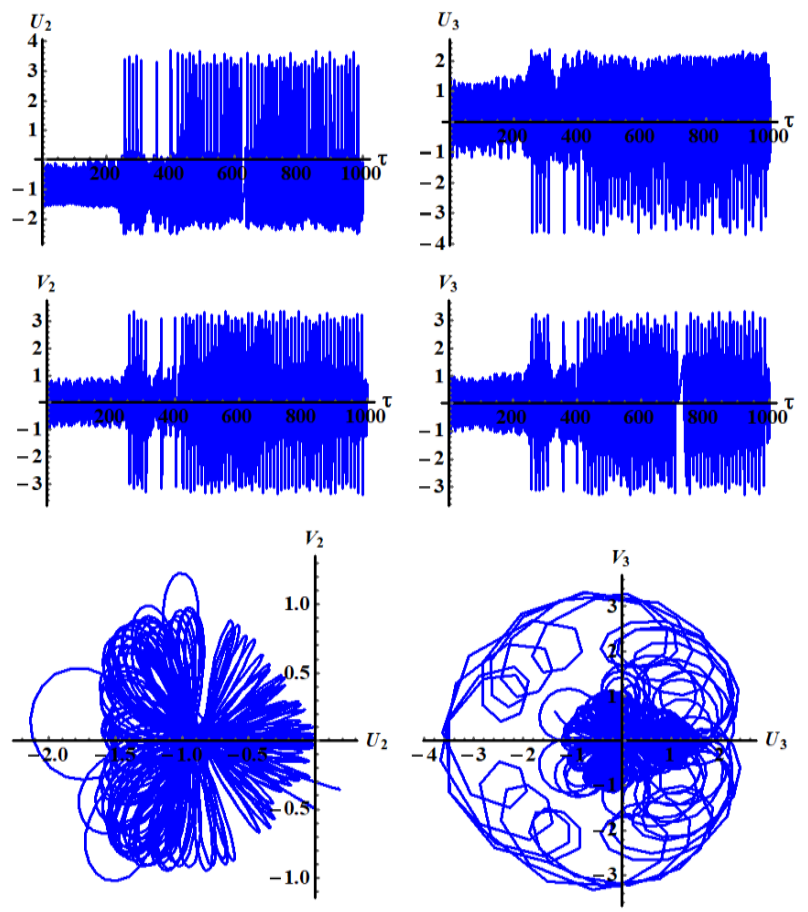

Fig.23. Displays the chaotic behavior of the considered system.

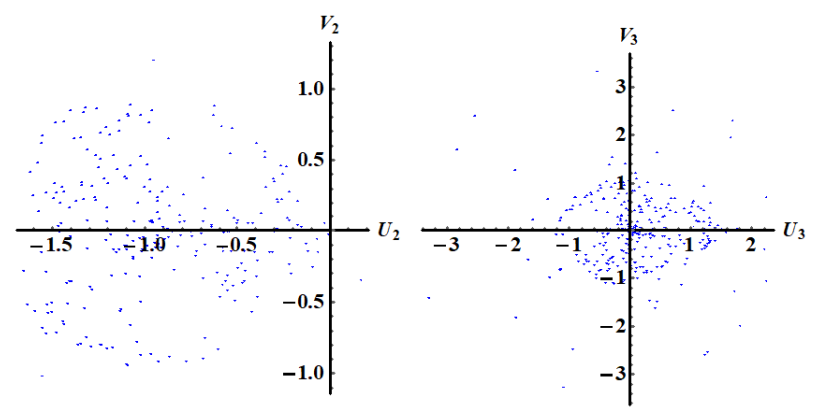

Fig.24. Poincarè map corresponding to chaotic motion.
An inspection of figures 14-16, 17-19, and 2022 show that, the modified phases and amplitudes increase in which the last ones have a periodic manner.

Also, we can observe that the chaotic motion in Figure.23 when the parameters take the following values:

$$
\begin{aligned}
& \sigma_{1}=-0.001, \quad \sigma_{3}=0.0205, \quad F_{0}=30 \mathrm{~N}, \\
& M_{1}=0.02 \mathrm{~N} . \mathrm{m}, \quad \Omega=0.2 \mathrm{rad} . \mathrm{s}^{-1}, \quad \varepsilon=0.005 \\
& \ell_{1}=10 \mathrm{~m}, \quad \ell_{2}=0.5 \mathrm{~m}, \quad \ell_{3}=0.8 \mathrm{~m}, \\
& m_{1}=10 \mathrm{~kg}, \quad m_{2}=5 \mathrm{~kg}, \quad m_{3}=8 \mathrm{~kg} .
\end{aligned}
$$

Figure.24 reveals the Poincarè map corresponding to the chaotic behaviour of the dynamical system. Such a map plots a point in the phase plane every period of the trajectory. However, if the dynamical system admits a periodic solution, the corresponding Poincarè map is represented by a point or series of points as the period is a multiple of the forcing. If the solution is quasi-periodic, the Poincarè map will be illustrated by orbit. This plot does not seem to have any significant structure, which indicates that the solution is truly chaotic.

\section{Conclusion}

This paper addresses the nonlinear dynamical motion of TP in which its pivot point moves in a circular path with constant angular velocity under the action of harmonic excitation force at the free end of TP and one harmonic moment at the point of suspension. The GEOM is derived using Lagrange's equations and is solved using MST to obtain the approximate solutions up to the third approximation. Resonance cases are classified, in which a combination of simultaneously primary external and internal resonance is investigated. The modulation equations are achieved given obtained solvability conditions. Time history plots of the obtained approximate solutions are presented, to describe the dynamical motion at any instant. Moreover, resonance curves are as well as the 
modified amplitudes were subsequently justified in whole time interval for different parametric values and the properties of the amplitudes are plotted in phase plane curves. The stability of the considered dynamical model applying the nonlinear stability analysis approach is checked. The attained results are considered as generalizations of those which were studied previously in (Gupta et al., 2016; Braun, 2003; Gupta et al., 2017) (for the case of fixed suspension point and in absence of external forces and torques).

\section{References}

Amer, T. S., Bek, M. A. \& Hamada, I. S. 2016. On the motion of harmonically excited spring pendulum in the elliptic path near resonances, Adv Math Phys, Volume 2016, 15 pages.

Amer, T. S., Bek, M. A. \& Abouhmr, M. K. 2018. On the vibrational analysis for the motion of a harmonically damped rigid body pendulum, Nonlinear Dyn 91, 2485-2502.

Amer, T. S., Bek, M. A. \& Abouhmr, M. K. 2019. On the motion of a harmonically excited damped spring pendulum in an elliptic path, Mech Res Commu 95, 23-34.

Awrejcewicz, J., Kudra, G. \& Lamarque, C. H. 2004. Investigation of a triple physical pendulum with impacts using fundamental solution matrices, Int J Bifur Chaos 14, 12, 4191-4213.

Awrejcewicz, J. \& Kudra, G. 2005a. The piston-connecting rod-crankshaft system as a triple physical pendulum with impacts, Int. J. Bifurc. Chaos 15, 2207-2226.

Awrejcewicz, J. \& Kudra, G. 2005b. Stability analysis and Lyapunov exponents of a multi-body mechanical system with rigid unilateral constraints, Nonlinear Analysis 63, e909-e918.
Awrejcewicz, J., Kudra, G. \& Wasilewski, G. 2007. Experimental and numerical investigation of chaotic regions in the triple physical pendulum, Nonlinear Dyn 50, 755776.

Awrejcewicz, J. 2012. Classical mechanics: Dynamics, Springer, Berlin.

Awrejcewicz, J., Starosta, R. \& Kamińska, G. 2013. Asymptotic analysis of resonances in nonlinear vibrations of the 3-of pendulum, Differ Equ Dyn Syst 21, 1\&2, 123-140.

Awrejcewicz, J., Starosta, R. \& Kamińska, G. S. 2016. Stationary and transient resonant response of a spring pendulum, Procedia IUTAM 19, 201-208.

Braun, M. 2003. On some properties of the multiple pendulums, Arch. Appl. Mech. 72, 899-910.

El-Sabaa, F. M., Amer, T. S., Gad, H. M. \& Bek, M. A. 2020. On the motion of a damped rigid body near resonances under the influence of harmonically external force and moments, Results in Physics 19, 103352.

Gupta, M. K., Sinha, N., Bansal, K. \& Singh, A. K. 2016. Natural frequencies of multiple pendulum systems under free condition, Arch Appl Mech 86, 1049-1061.

Gupta, M. K., Sharma, P., Mondal, A. \& Kumar, A. 2017. Visual recurrence analysis of the chaotic and regular motion of a multiple pendulum system, Arab J Sci Eng 42, 27112716.

Jaafar, H. I., Mohamed, Z., Shamsudin, M. A., Mohd Subha, N. A., Ramli, L. \& Abdullahi, A. M. 2019. Model reference command shaping for vibration control of multimode flexible systems with application to a double-pendulum overhead crane, Mech Syst Signal Process 115, 677-695. 
Kwiatkowski, R., Hoffmann, T. J. \& Kolodziej, A. 2017. Dynamics of a double mathematical pendulum with variable mass in dimensionless coordinates, Procedia Engineering 177, 439-443.

Kamińska, G., Starosta, R. \& Awrejcewicz, J. 2018. Two approaches in the analytical investigation of the spring pendulum, Vib Phys Syst 29, 2018005.

Nayfeh, A. H. \& Mook, D. T. 1979. Nonlinear Oscillations, Pure and Applied Mathematics, John Wiley \& Sons, New York.

Nayfeh, A. H. 2004. Perturbations methods, Wiley, Weinheim.

Plissi, M. V., Torrie, C. I., Husman, M. E. H., Robertson, N. A., Strain, K. A., Ward, H., Lück, H. \& Hough, J. 2000. GEO 600 triple pendulum suspension system: seismic isolation and control, Rev. Sci. Instrum. 71(6), 2539-2545.

Rajasekar, S. \& Sanjuan, M. A. 2016. Nonlinear resonances, Springer, Berlin.

Raymond, H. P. \& Virgin, L. N. 2013. Pendulum models of ponytail motion during walking and running, J Sound Vib 332, 3768-3780.

Starosta, R., Kamińska, G. S. \& Awrejcewicz, J. 2011. Parametric and external resonances in kinematically and externally excited nonlinear spring pendulum, Int $\mathbf{J}$ Bifurcat Chaos 21, 10, 3013-3021.

Starosta, R., Kamińska, G. S. \& Awrejcewicz, J. 2012. Asymptotic analysis of kinematically excited dynamical systems near resonances, Nonlinear Dyn 68, 459-469.

Vakakis, A. F.; Gendelman, O. V.; Bergman, L.A.; McFarland, D. M.; Kerschen, G. \& Lee, Y. S. 2009. Nonlinear targeted energy transfer in mechanical and structural systems, Springer Science Business Media, B.V.

Wolfram, S. 2017. An elementary introduction to the Wolfram Language, Wolfram Media; 2nd. Edition.

Submitted: $\quad 08 / 06 / 2020$

Revised: $\quad 14 / 09 / 2020$

Accepted: $\quad$ 18/10/2020

DOI: $\quad 10.48129 /$ kjs.v48i4.9915 